\title{
MECCA: Hypermedia Capturing of Collaborative Scientific Discourses about Movies
}

\author{
Ralf Klamma, Marc Spaniol, and Matthias Jarke \\ Lehrstuhl Informatik 5, RWTH Aachen University, Germany
}

klamma@cs.rwth-aachen.de mspaniol@cs.rwth-aachen.de jarke@cs.rwth-aachen.de

\begin{abstract}
The success of collaborative hypermedia systems in business, engineering, or humanities heavily depends on the discursive nature of knowledge creation. Informing systems that assist crossdisciplinary communities of practice in these fields should therefore be able to capture, to visualize, and to support the ongoing scientific discourse to keep participants informed and committed to the knowledge creation process. We present a solution for this issue, using the MECCA discourse support system for a movie research community as an example. MECCA integrates research processes with teaching processes in the humanities. Our study demonstrates that knowledge creation discourses involve a lot of re-“writing” (transcription) of discourse artifacts within or across media. We introduce an underlying formal technique to support flexible and adaptable transcription on hypermedia artifacts in the community. Our approach includes a linkage of knowledge to action which aims at seamless de-contextualization from action and recontextualization into action.
\end{abstract}

Keywords: Hypermedia, discourse support, humanities, MPEG-7, CSCL.

\section{Introduction}

Foucault defines 'discourse' as the medium by which ideas are exchanged (Wikipedia, 2004). Scholarly communication in the Humanities and the Cultural Studies heavily depend on the discursive nature of knowledge creation. Media changes over the centuries altered not only the communicational culture of scholars regarding their archives and text production strategies but also the communication situation in society, thus causing scientific, artistic, and societal paradigm shifts.

The DFG-funded interdisciplinary Collaborative Research Center "Media and Cultural Communication" was founded in 1999 to study the nature and impact of media use and change on the discourses in cultural and scientific communication.

Supported by historical analysis of media use, our project within this Center intends to create new

Material published as part of this journal, either on-line or in print, is copyrighted by the publisher of the Informing Science Journal. Permission to make digital or paper copy of part or all of these works for personal or classroom use is granted without fee provided that the copies are not made or distributed for profit or commercial advantage AND that copies 1) bear this notice in full and 2) give the full citation on the first page. It is permissible to abstract these works so long as credit is given. To copy in all other cases or to republish or to post on a server or to redistribute to lists requires specific permission and payment of a fee. Contact Editor@inform.nu to request redistribution permission. workplaces for scientists in the 21st century. These interdisciplinary workplaces will influence the way scientists organize knowledge and cooperation within networked hypermedia information systems. Research and results will also have impact on the organization of social, economic and politic knowledge organization and cooperation patterns like the organization of public debates. 
Supporting communication and knowledge creation in digitally networked cultural science communities represents a specific challenge for the development and organizational structure of hypermedia information systems. Several years of cooperation with scientists from different disciplines in the humanities and the cultural studies have revealed at least three main requirements:

1. Semantic Freedom: The discursive nature of knowledge creation in the humanities, based on an intense exposure to hypermedia artifacts and underlying theories, requires support of different digital media to be combined with (almost) arbitrary metadata which characterize the situational background of an artifact. These digital artifacts are reifications of actual discourses and have special semantics for each user and each community of practice (Wenger, 1998). The situational backgrounds belonging to an artifact can be quite different depending on the scholar, her role in the community and her educational background. Concepts for dynamic hypermedia context management with a high degree of semantic freedom are thus needed for scholarly communications in the humanities.

2. Openness of repositories: This is an indispensable prerequisite for scaling such systems beyond the purposes of one community of practice. Scientific discourses in an informed society could not be isolated in the ivory tower anymore. Interdisciplinary and networked work settings lead to more exchange within the scientific community as well with society, industry and the political system. Modern information system should allow scholars of any kind to check out and modify archives, and bring them back into the discussion, again fostering discourses by simultaneously assuring intellectual property. We are aware that this is not only a requirement of a class of systems but is accompanied by the transformation of the humanities themselves.

3. Fluidity of archives: A naive multimedia understanding - e.g. that hypermedia can transport knowledge - is insufficient, since complex interrelations between media exist as well as complex cultural interfaces to these media (Manovich, 2001). Multimedia repositories are a big advantage but the contextualized and meaningful management of hypermedia artifacts needs more advanced hypermedia management strategies (fluid archives) than the usual basic support for storage and retrieval on the basis of low-level feature descriptors implementing old-fashioned de-contextualization strategies propagated in stateof-the-art information systems for scholarly communications.

In the remainder of this paper, we discuss related literature first. We introduce a theoretical reflection on knowledge creation processes in the humanities related to the use of different media. Based on this, an analysis of general requirements for hypermedia discourse support systems supporting the three concepts semantic freedom, scalable repository support and fluidity of archives follows in section "Supporting Scholars within Scientific Discourses in the Humanities". We then analyze Existing Approaches to Support Discourses

in the Humanities to have a sound basis for our implementation. To address the conflict between total freedom and fluidity on the one hand and the needs for a formal basis for advanced management concepts, we discuss Repository Techniques to Capture Semantics of Multimedia Artifacts separately.Finally, we present an in-depth analysis for the case study of our Movie E-learning Combination and Categorization Application (MECCA) that aims at fostering scholarly communications in the film studies. The paper closes with conclusions and an outlook on further research.

\section{Related Work}

While the idea of a technical implementation of hypertext is quite old (Bush, 1945), the idea was renewed by Ted Nelson in the 1960's and implemented in the Xanadu prototype (http://xanadu.com/) (Nelson, 1999). He coined the term "hypertext” and wrote (Nelson, 1967): 
Hypertext is the combination of natural language text with the computer's capacity for interactive branching, or dynamic display, when explicitly used as a medium. Or, to define it more broadly, 'hypertext' is the generic term for any text which cannot be printed (or printed conveniently) on a conventional page, or used conveniently when bound between conventional covers. 'Non-linear text' might be a fair approximation.

The most important concepts of hypertext are therefore non-linearity of text, computer supported links and the interactive way of using hypertext. Thus, 20 years later, Jeff Conklin reduced the technical definition of hypertext to (Conklin, 1987):

The concept of hypertext is quite simple: Windows on the screen are associated with objects in a database, and links are provided between these objects.

Objects can contain any digital data, text or image. These data-filled objects will be referred to as "hypermedia artifacts" below. In the Humanities the Apple Hypercard was a very successful tool which transformed millions of slip boxes into hypertext. In modern hypertext or hypermedia (Garzotto, Mainetti \& Paolini 1995; Nelson, 1999) so different systems issues like adaptability (Brusilovsky, 1996), multiculturalism (Scollon, 1999) or multimodality (Lemke, 2002) are discussed in the frame of an emerging theory of multimedia semiotics (Daft \& Lengel, 1986). Multimedia discourse support systems for scholarly communications exist for many application domains like scholarly publishing (Buckingham Shum et al., 1996), software engineering (Kaplan, Tolone, Carroll, Bogia, \& Bignoli, 1992; Sumner \& Buckingham Shum, 1998), dancing studies (Golshani \& Vissicaro, 2002), image collections (Grosky \& Sreenath, 2001), video databases (Carrer, Ligresti, Ahanger, \& Little, 1997; Dönderler, Saykol, Ulusoy, \& Güdükbay, 2003; Smith, Blankinship \& Lackner, 2000), and help systems (Ackerman \& McDonald, 1996). There is still an ongoing discussion between the hypermedia community and the web community if hypermedia systems and web-based system can solve the same problems (Berners-Lee, 2003). Clearly, scalability issues are addressed more deeply in the web-based system community (Lennon \& Maurer, 1996).

Learning from discourses in a community was discussed in a more user-centric manner by the concept of cognitive apprenticeship (Collins, Brown \& Newman, 1998; Lave \& Wenger, 1991) before the community itself came into the focus of research with the seminal book of Etienne Wenger (Wenger, 1998). Several hypermedia systems for computer supported collaborative learning (CSCL) were built in the 1990's (Brown, Ash \& Rutherford, 1993; Bruckman , 1994; Jonassen \& Mandl, 1990; Jonassen et al., 1997; Ligorio, 2001; Looi \& Ang, 2000; Preston \& Wilson, 2001; Rodriguez, Silva, Rosano, Contreras, \& Vitela, 2001; Schär \& Krueger, 2000), many of them in a constructivistic setting (Bruckman , 1998; Duffy \& Jonassen, 1992; von Glasersfeld, 1996). Goal of CSCL is to create common knowledge by integration of different user perspectives (Pfister, Wessner, Holmer, \& Steinmetz, 1999) and by promoting sharing and discourses (Miao, Fleschutz \& Zentel, 1999).

Learning repository support (Dhraief, Nejdl, Wolf, \& Wolpers, 2001; Dumas, Lozano, Fauvet, Martin, \& Scholl, 2000; Fendt, 2001; Henze, 2000; Hunter, 2001b) was developed in the last years with a focus on metadata standards such as Dublin Core (Hillmann 2001), RDF (Bray, 1998; „World Wide Web Consortium: Resource Description Framework (RDF)“, 1999), MPEG7 (Salembier \& Sikora, 2002) and ontology management (Berners-Lee, Hendler \& Lassila 2001; Gomez-Peres, Corcho, 2002).

In computer science, an ontology is considered a specification of shared conceptualization (Gruber, 1993) within a ongoing discourse. The formal knowledge representation language OWL (Smith, Welty \& McGuinness, 2004) is used to specifiy ontologies and inference mechanisms in the semantic web (Berners-Lee, Hendler \& Lassila, 2001). 
The XML-based metadata standard MPEG-7 has been introduced by the moving pictures expert group (MPEG) (van Beek, Yoon \& Ferman, 2002). While MPEG-1, MPEG-2, and MPEG-4 present the multimedia information itself, MPEG-7 offers means to store information about the information („the bits of the bits“). Being independent of the media storage format, MPEG-7 offers the most comprehensive pallet of descriptions for audio-visual materials on different levels of granularity (Hunter, 2001a; Kosch, 2002). Core elements of MPEG-7 are:

- Descriptors, characterizing syntax and semantics of the different features of audio-visual materials.

- Description Schemes (MDS), describing structures and relations between elements and schemes.

- Description Definition Language, (DDL), a common language to create or extend descriptors and description schemes.

MPEG-7 documents are represented in XML. The MPEG-7 syntax is determined by the DDL „XML Schema Definition Language“ (XML Schema). The Multimedia Description Schemes (MDS) are at the core of the MPEG-7 standard constituting a library of descriptors and description schemes to store metadata about multimedia materials. The MDS are divided into functional areas visualized in Figure 1.
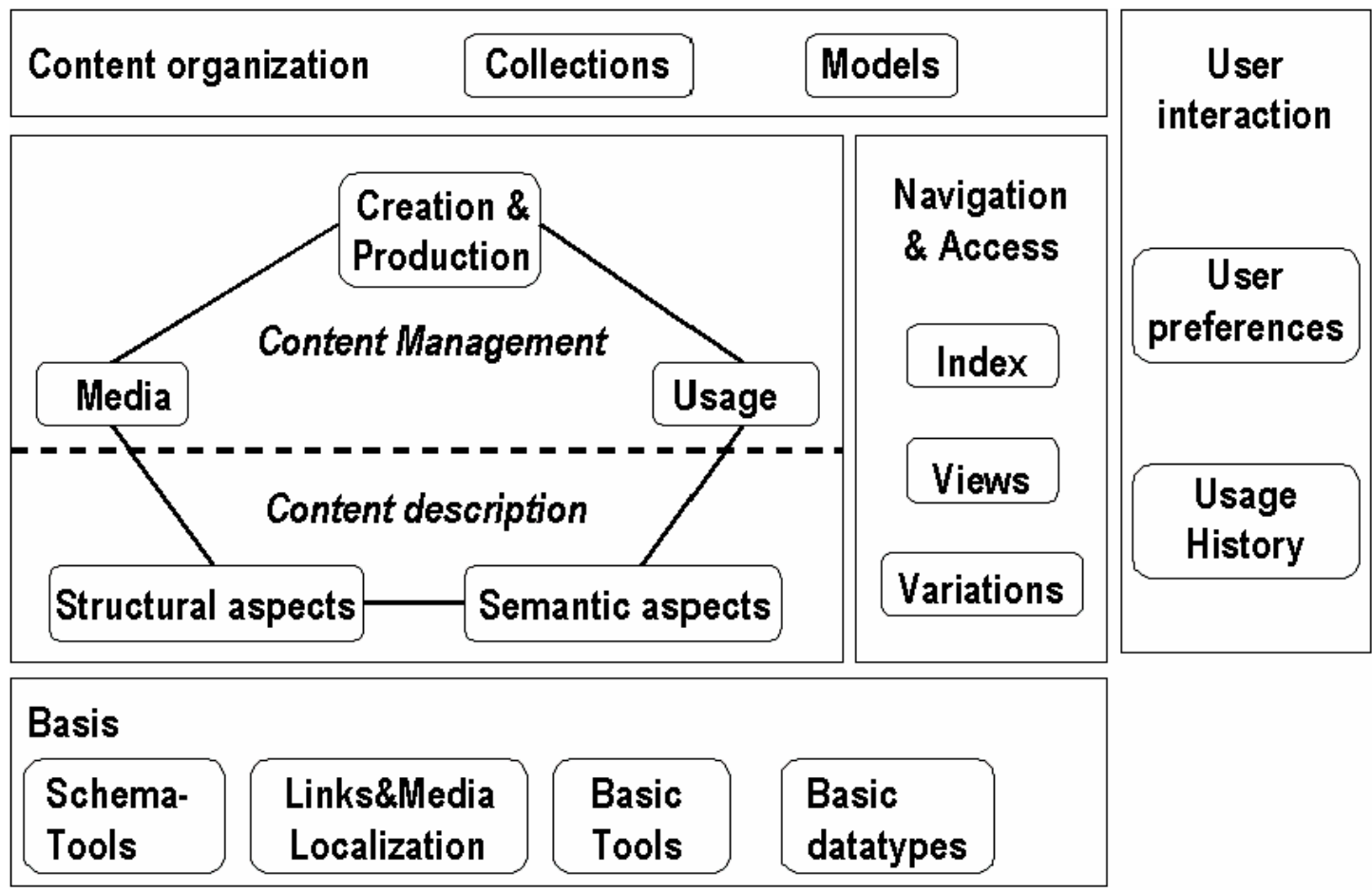

Figure 1: Overview on features provided by MPEG-7

Each basic element contains specific descriptors and data types that are usable in all MPEG-7 schemes and therefore, are a flexible and powerful tool for the creation of metadata. In the core of the MDS are the description of the content of multimedia materials within „Content Management \& Description“. „Creation \& Production“, „Media Information“, and „Usage Information“ structure information about the product life cycle of multimedia artifacts, e.g. producer, rights information, clients constraints. „Structural Aspects“ and „Conceptual Aspects“ describe the spatial, 
temporal or spatio-temporal segmentation of the artifacts and the conceptual segmentation into abstract objects, agents, events, places and points in time in the narrative world of the medium. Different “Summaries” of audio-visual materials are possible in „Navigation \& Access“. „Views“ and „Variations“ facilitate different accessible versions. „Content Organization“ is used for collections and analytical or statistical models. Personalized access on multimedia artifacts is defined with scheme „User Interaction“. Usage history and preferences of scholars can be managed within the scheme.

Experiences with earlier multimedia standards like Dublin Core lead to the insight that the effort of harmonizing relatively small ontologies often appears frightening and generates questions about scalability (Doerr, Hunter \& Lagoze, 2003). Surely, there is no alternative to merging of ontologies, which raises new scientific challenges. It considers intellectual effort and is a learning process for individuals as well as their communities. Basically, core ontologies are small and give an opportunity to specify a larger amount of specialized concepts (Guarino, 1998). Special importance for discourse analysis has the detection of similarities in the use of a certain vocabulary by different users (Miller \& Charles, 1991; Noy \& Musen, 2002; Resnik, 1998; Rodriguez \& Egenhofer, 2003; Tversky, 1977). A detailed overview about metadata support for scalable repositories can be found in (Jarke, Klamma \& Lyytinen, 2005; Westermann \& Klas, 2003).

\section{A Transcriptive Theory for Discourses in the Humanities}

For all the many facets of networked information systems the general thesis is that successful communication and knowledge organization in the Humanities depend on the choice or even the construction of appropriate media for specific scientific discourses. The underlying basic relation between knowledge organization and communication is the so-called Principle of Transcriptivity (Jäger \& Stanitzek, 2002) for the intra- and inter-media reading and producing of media artifacts (cf. Figure 2), allowing the following operations:

1. Transcriptions condense and structure the set of pre-"texts" by designating some of them as evidences, counter-examples, or critique, while de-emphasizing others.

2. Transcriptions thus enable a new reading of the pre-“texts“; they also determine which readershipthey address, by the media through which the transcript is prepared and communicated. So, design of transcripts can significantly increase the number of addressees for a certain artifact, or may intentionally focus on specific 'insiders'.

3. Thus, transcription does not only change the status of passive understanding by other scholars, but it also enables further cooperative knowledge creation by stimulating debate about the selected pre-texts and about the transcription itself.

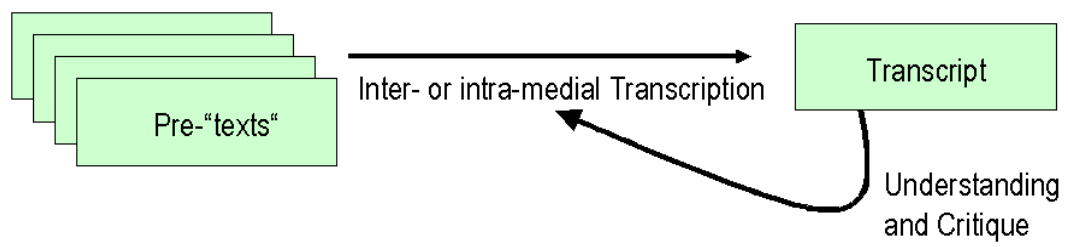

Figure 2: The principle of transcriptivity [adopted from (Jäger, 2002)]

As a prototypical example (Jäger, 2002), consider a historical narrative which sheds provocative new light on the almost infinite set of historical records, thus causing a debate including criticisms of both the chosen sources and the chosen transcription. Transcriptions in constructed media can radically change the structure of the community of practice. As an example, a Judaistic hypothesis of how discourse knowledge was encoded within Talmudic tractates over its development history of several centuries, has been the basis for a transcription of the traditional Mishna 
and Gemara Tora rolls into a structured and annotated multi-lingual hypertext using XMLoriented metadata management (Hollender, Klamma, Börner-Klein, \& Jarke, 2001; Klamma, Hollender, Jarke, Moog, \& Wulf, 2002). This transcript, for example, supports switching between languages such as English, German, and Hebrew while maintaining the discourse structure through e.g. color highlighting and annotations. Such features make these texts - formerly readable only by a few rabbinic specialists - accessible to beginning students and other interested scholars with limited knowledge in both Hebrew and Judaistic concepts. This re-addressing has rapidly created a worldwide teaching and learning community (of course including heated debates about the adequacy of the underlying theory itself).

We have to emphasize the careful design of media in addition to underlying formalism, including attention focusing by suitable transcription techniques to build successful hypermedia discourse support systems. However, only in limited and well-managed settings such as data warehousing, current transcription mechanisms involve rich semantic or media transformations, powerful quality checks, and the like.

\section{Supporting Scholars within Scientific Discourses in the Humanities}

In scientific discourses, different kinds of scholars involved form a community of practice. Communities of practice (CoP) are characterized by common conventions, language, tool usage, values, and standards (Wenger, 1998). The development of a common practice which defines the community comprises the negotiation of meaning among the participants as well as the mutual engagement in joint enterprises and a shared repertoire of activities, symbols, and artifacts. A $\mathrm{CoP}$ is inseparable from issues of (individual and social) identity. Identity is mainly determined by negotiated experience of one's self in terms of participation in a community and the learning process concerning one's membership in a CoP (Wenger, 1998). The approach combines the "two sides of the medal" of community participation: the social practice of the community as a collective phenomenon, and the identity of its member scholars as an individual one. Learning is the permanent construction of knowledge, based on former experiences (Lave \& Wenger, 1991).

In our case, the scientists are the most important scholars, creating the knowledge through their original research. Students and assistants are participating in a legitimate peripheral manner (Lave \& Wenger, 1991). Multipliers such as publishers, journalists, and the interested public spread the results of discourses into other discourses, whereby it is the ultimate goal of the Humanities to keep the discourses open for new ones. While a few systems have been built and used successfully within a single CoP (cf. section 2), discourse support across disciplines and translation of vocabularies to lower barriers of discourse participation are still open issues. Specifically, discourses are constructing the things they talk about in the discourse itself (Foucault, 1982). This highly dynamic situation is a grand challenge hypermedia or web-based discourse support systems. Because we support many kinds of scientific discourses, we use the terms knowledge creation and learning often as synonyms.

Systems should aim at providing scholars with a flexible (online) environment to create, annotate and share media-rich documents for the discourses, by relying on meta data standards. That allows scholars to create and exchange multimedia artifacts and collections of them in communities across disciplines and distances by collaborating on multimedia artifacts. Thus, media literacy skills are improved and media influences are understood better, enhancing skills at communicating effectively in today's increasingly global world of science, education, and business.

We now discuss requirements for hypermedia systems in $\mathrm{CoP}$ in the humanities on an abstract but technical level. These requirements follow our discussion in the introduction about semantic freedom, the openness of repositories and the fluidity of archives. The main purpose of these re- 
quirements is to compare different approaches within the last years to which extent they meet the listed requirements. Furthermore, we will discuss how our own case study meets the requirements at the end of the paper.

Collective hypermedia artifact repository. Scientists, teachers, students and other scholars need access to digital archives on several levels through one interface to archive a variety of digital formats and content types. Each artifact needs to be tagged and managed by textual meta data. Textual meta data may be high-level descriptions but also low-level features. Applications compliant to standards may exchange media files and their meta data. An integrated permission management allows users to share materials via the repository, encouraging constructivist models of learning and research, by simultaneously protecting their intellectual property. Distribution of repositories is possible in different scenarios like peer-to-peer.

Transcription. Semantic enrichment of data and usage of other file formats or even media for a better understanding is closely connected to the discursive and transcriptive process of knowledge creation. In the hypermedia repository the traceable creation of new links or tags can be conceptualized as transcriptive behavior enhancing the readability of hypermedia collections. Collaborative learning is encouraged by annotations having to be accessible and possibly transcribed by other scholars. Here, there is a particular necessity to combine primary and secondary practices of hypermedia artifact management where primary practices address the creation of multimedia artifacts and secondary practices addresses the re-organization and re-use of artifacts in new discourses..

Search and retrieval. The creation of hypermedia artifacts needs meta data annotations in a flexible and open schema to be stored in a repository for a latter retrieval. To be fully aware of hypermedia semantics we have to reflect the transcriptive knowledge creation process which means that all processes of retrieval, manipulation, management should be accessible as objects in the repository, too. Moreover, tools to understand other scholars' views by bringing artifacts in another situational context are needed.

Community management. Scientific discourses are practices of high relevance both for scientists and students participating in a legitimate peripheral manner. Discourses should give even locally distributed CoP a community-like access to the hypermedia information systems. CoP need a flexible user management with access rights on different levels and with different roles.

Personal and group collections. Sharing knowledge with others means storing and retrieving hypermedia artifacts from a collective repository. Since humanities subjects often rely on collections, there is a need for fluid archives on personal and group level, which can be navigated, sorted, and annotated by community members. In our sense, fluid archives are created at a particular point of time and in a specific context, which underlie an ongoing discourse and have to be (re-)contextualized continuously. A variety of navigation interfaces for archives is needed, by simultaneously offering a standardized backbone among all systems.

Hypermedia and interrelation graphs. Knowledge depends on the medium through which it has been communicated. So, a singular artifact lacks its context. To express their full context and complexity, scholars have to modify interrelations of hypermedia by hypermedia graphs, and to comment on them. The visual representation of knowledge is important to express media dependencies, which helps overcoming gaps from (mis-)interpretation of artifacts when exchanged via a collective repository.

Ontologies. Ontologies are quite popular within the knowledge engineering community for structuring content. They are commonly applied in information brokering and provide users with content esteemed the most suitable in a particular context. The problem is that hypermedia semantics - a not necessarily shared set of interpretative acts within a CoP - are very hard to capture either 
manually or automatically. In addition, the discursive act of knowledge creation in the humanities contains an inherent need for a modification (transcription) of different media in an arbitrary and interchangeable manner.

We want to point out that only a deep analysis of a $\mathrm{CoP}$ and the reflection of communications taking place may lead to information systems that are able to disclose non-trivial technical and CoP-aware requirements for open, scalable, and fluid hypermedia discourse support systems in the Humanities. We now present some existing approaches in the field of discourse support in the Humanities. Thereby, we examine the management strategies to handle hypermedia artifacts in more detail and afterwards discuss the pro and cons of these approaches with respect to their usefulness in supporting discourses of $\mathrm{CoP}$ in the humanities.

\section{Existing Approaches to Support Discourses in the Humanities}

Many different systems have been successfully created for isolated research groups or curricula in the Humanities. However, cross-disciplinary or even public use of these systems for research, learning, or debating purposes is rare. Based on our setting, only a few approaches can be compared with our approach on supporting discourses in cross-disciplinary CoP.

\section{Warburg Electronic Library (WEL)}

The WEL for political iconography was developed in cooperation of M. Warnke (Uni Hamburg) and J.W. Schmidt (TU Hamburg-Harburg). Political iconography basically intends to capture the semantics of key concepts of the political realm under the assumption that political goals, roles, values, means etc. require mass communication which is implemented by the iconographic use of images (Schmidt, Sehring \& Warnke, 2001).

The WEL represents a media transition from traditional slip boxes to hypermedia structures in digital media. The core is a card index, which consists of cards containing what researchers have defined as representative samples of political concepts. The repository is constructed after the principles of the cultural scientist Aby Warburg (1866-1929). Warburg built up a huge library for the cultural sciences (33.000 books) organized by the principle of good neighborhood. The Warburg Institute in London writes on the webpage (http://www.sas.ac.uk/Warburg/default.htm):

Thus he conceived the programme of illustrating the processes by which the memory of the past affects a culture. The paradigm he chose was the influence of antiquity on modern European civilization in all its aspects - social, political, religious, scientific, philosophical, literary and artistic - and he ordered his private library in Hamburg accordingly.

The project is an excellent example for the media change from the traditional letter case to a hypertext system. The original letter case in the Aby Warburg House in Hamburg was developed by the art historian Martin Warnke and it is his own intellectual property. The WEL prototype "allows interdisciplinary experiments and insights into multimedia content management and applications”. However, it transfers the intellectual property of M. Warnke to the Internet. Students and researchers are allowed to take image cards out of the index but never can put new cards into the index. We have analyzed an Art History seminar which used the WEL prototype to take out handset for the students. The students organized the picture cards in the handset and new materials from our source to create a multimedia essay on different topics like "Isabella d'Estes Studiolo and Grotta” (http://www.sts.tu-harburg.de/projects/WEL/welkga/themugr.htm). The presentation was done as a website using HTML and advanced digital image processing software reconstructing the rooms virtually (cf. Figure 3). But at the end of the seminar none of the materials collected by the students made it into the index because it was technically impossible to differen- 
tiate between M. Warnke's original contribution to the index and newly created stuff for the electronic version of the index only.

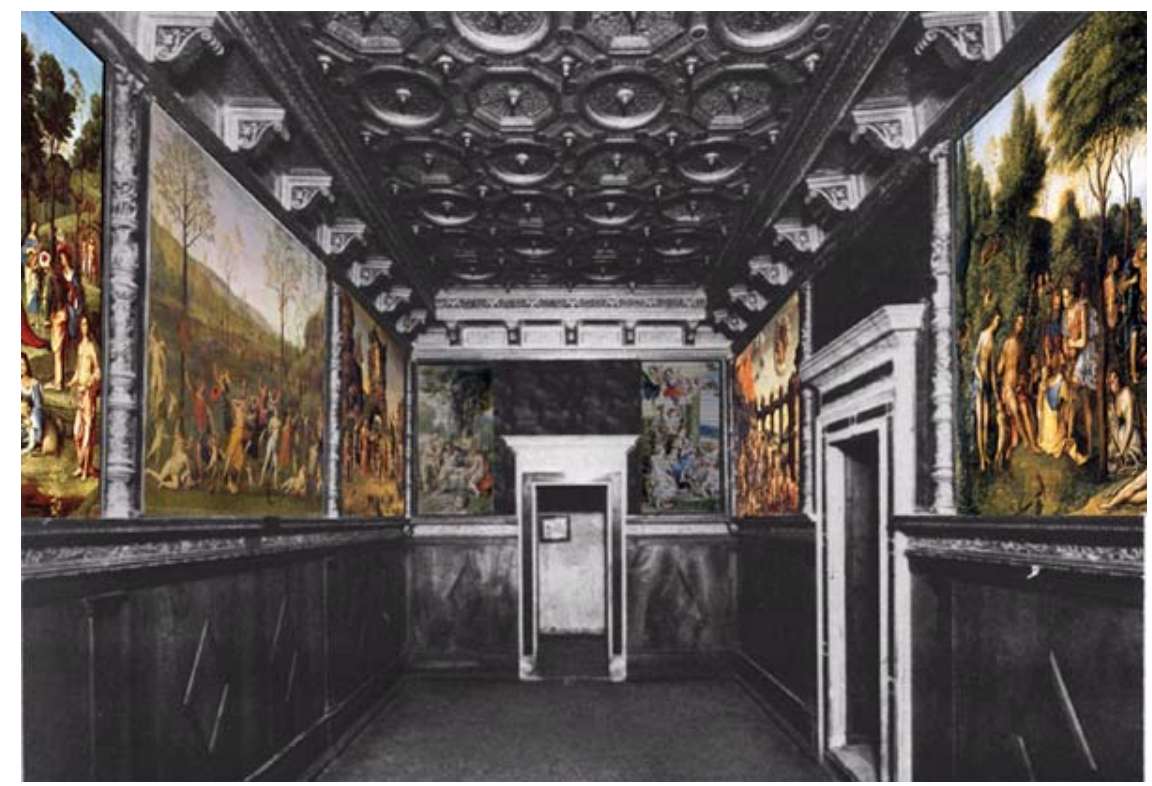

Figure 3: The virtually reconstructed studiolo

\section{Comprehensive Electronic Study Environment (CESE)}

The Babylonian Talmud is one of the favorite examples of existing hypertexts before hypertext theory. It is the largest collection of texts from rabbinic literature, covering some 5000 folio pages (cf. Figure 4) in the standard edition printed by Romm in Vilna (1880-1886). Its traditional layout has invited many interpretations both from scholars of Jewish Studies and from hypertextspecialists and scholars of literary hypertexts. D. Porush notes (Porush, 1994):

The Talmud is just as likely to "send" the reader to a page elsewhere in the Talmud as to the next page. You can open the Talmud almost anywhere to begin, although standard Talmudic learning progresses, at least at first, in a highly-arbitrary sequence of books. We also see that the Talmud promotes marginalia, scribbling or commentary, and a nonlinear non-directed form of knowledge. ... Notes and marks refer the reader to arguments elsewhere in the Talmud. In short, modern computer users will quickly recognize that the Talmud is a hypertext system: a means of gathering clusters of information that is nonlinear, promotes interpretation, is multi-vocal (or collaborative in the extreme), tends towards anonymity, is non-directed, packages information in multi-referential but discrete pages, and de-constructs the authority of a single author.

CESE is a joint product of the Jewish Studies (Universities of Cologne, Duisburg, and Düsseldorf) and Computer Science (RWTH Aachen) within the Collaborative Research Center "Media and Cultural Communication”. CESE is a hypertext learning environment for the Jewish studies (Hollender et al., 2001). By stressing the visualization of the parts of the text that can be studied independent of the specific topic discussed, and by enriching it with on-demand accessible information on the rules that govern their respective uses, readers who are not familiar with rabbinic literature may faster develop skills needed to read, study and understand. CESE was used in university-level teaching at the universities of Duisburg and Düsseldorf. 

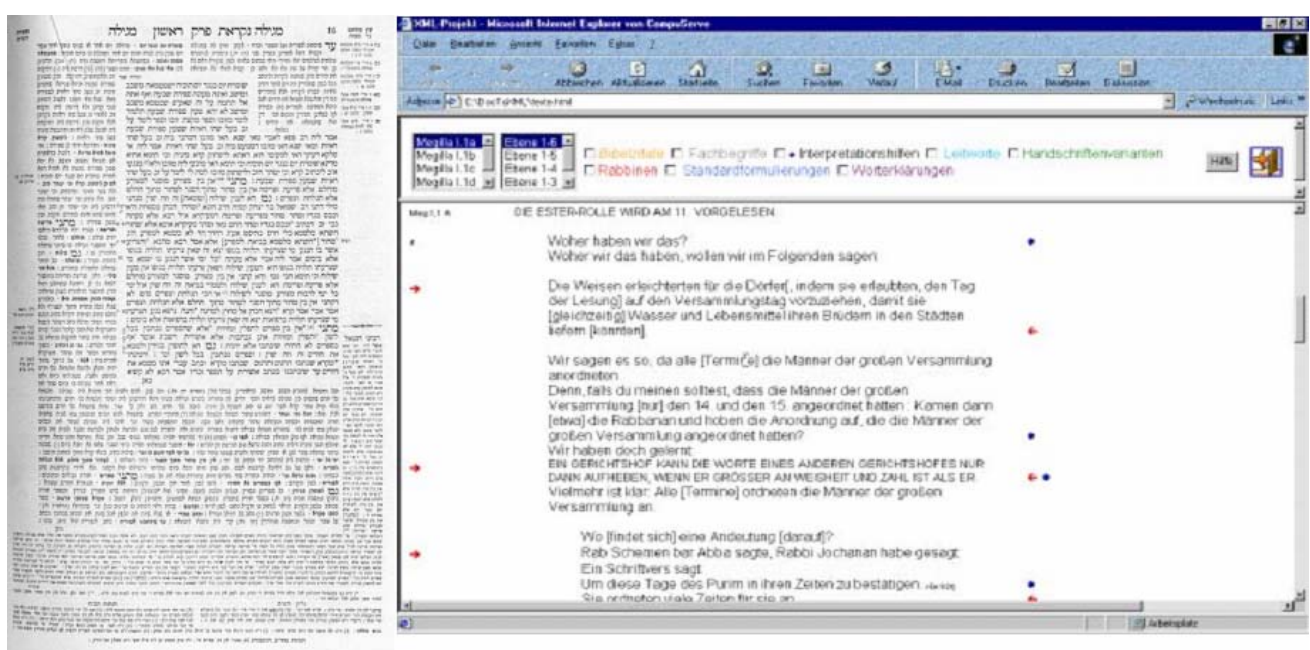

Figure 4: Folio page of the Babylonian Talmud and transcription in CESE.

\section{Berliner sehen}

"Berliner sehen" is a hypermedia documentary for German Studies developed at MIT that relies on an extensive collection of shared archives and the Internet to form a collaborative learning environment for beginning to advanced-level students (Fendt, 2001). Focusing on Berlin, this documentary features live-recorded video and authentic historical documents that depict the cultural, social, and political life of the city.

The hypermedia format of "Berliner sehen" encourages students to investigate material in context from different perspectives, to create their own hypermedia mini-documentaries, and to collaborate with other students on the expansion of the archives (cf. Figure 5).

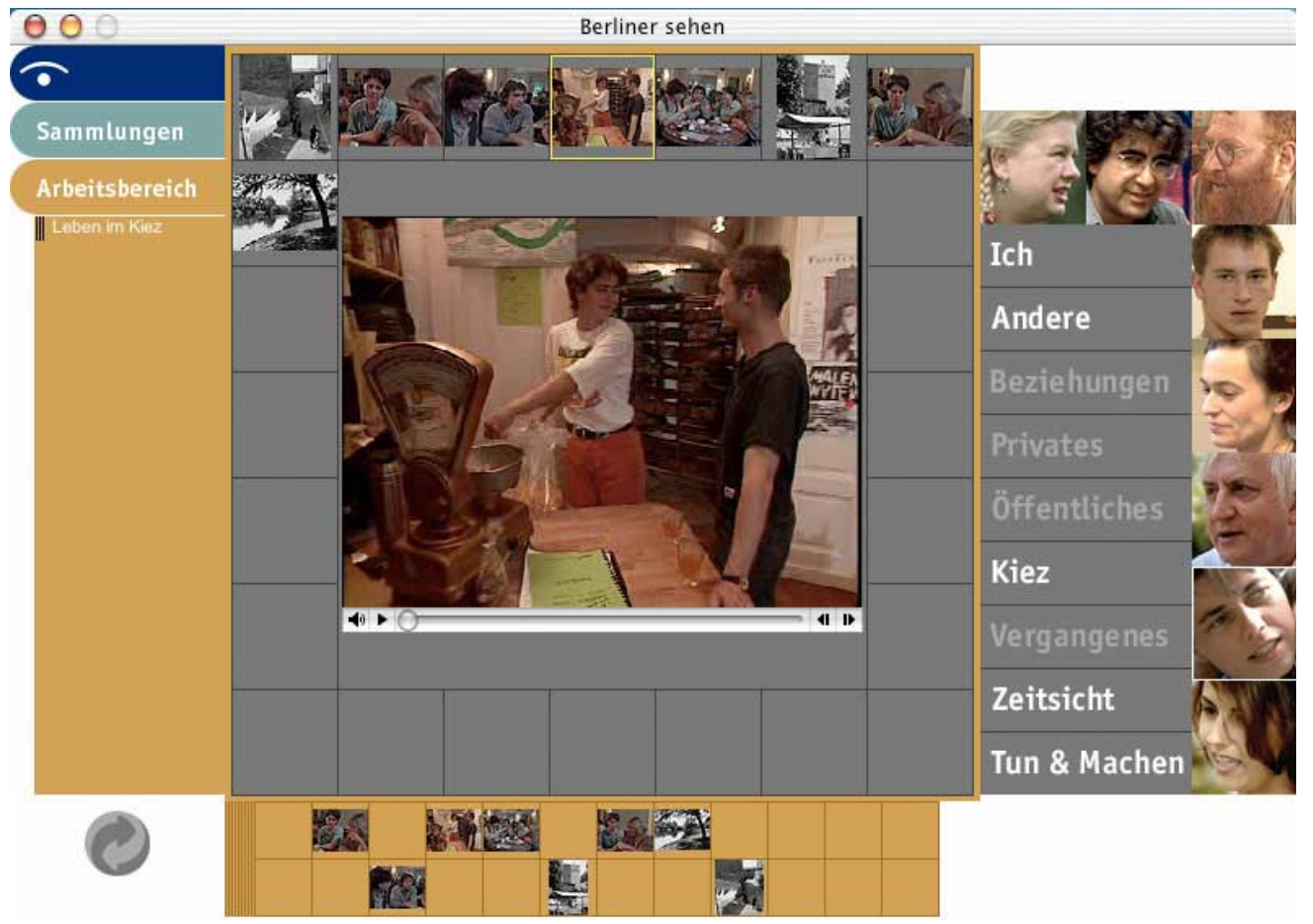

Figure 5: Front end of "Berliner sehen" 
The contemporary video core of "Berliner sehen" consists of several hours of natural conversations with Berlin residents from different social backgrounds. Spoken in authentic German, they acquaint students with the many facets of individual lives. Together with the extensive archive of texts, images, historical audio and video, these conversations form an expansive narrative network that engages students in exploring key cultural issues from diverse points of view. The footage for "Berliner sehen" was filmed during Summer 1995 by the Berlin-based German documentary video artists INTERACT, who worked in close collaboration with project directors Crocker and Fendt to create a video expressly designed for the hypermedia format of this project.

\section{Journal of Interactive Media in Education (JIME/D $\left.{ }^{3} E\right)$}

JIME is a freely available e-journal, published since 1996, targeting at researchers and practitioners interested in educational technology (Buckingham Shum \& Summer, 2001). JIME was founded with three goals in mind. First, it is intended to be a forum for innovative work in its field. Second, readers should be empowered to directly experience the systems and techniques being described. Third, it is driven by the hope that progress in multidisciplinary field might be best advanced by bringing together people reflecting the field's multiplicity of perspectives. Of particular concern is the persistent gap between educational theory and educational practice, wherefore one of the major goals is to bridge the disciplinary and experiential gaps by fostering dialogue between participants from diverse backgrounds and distant geographic locations.

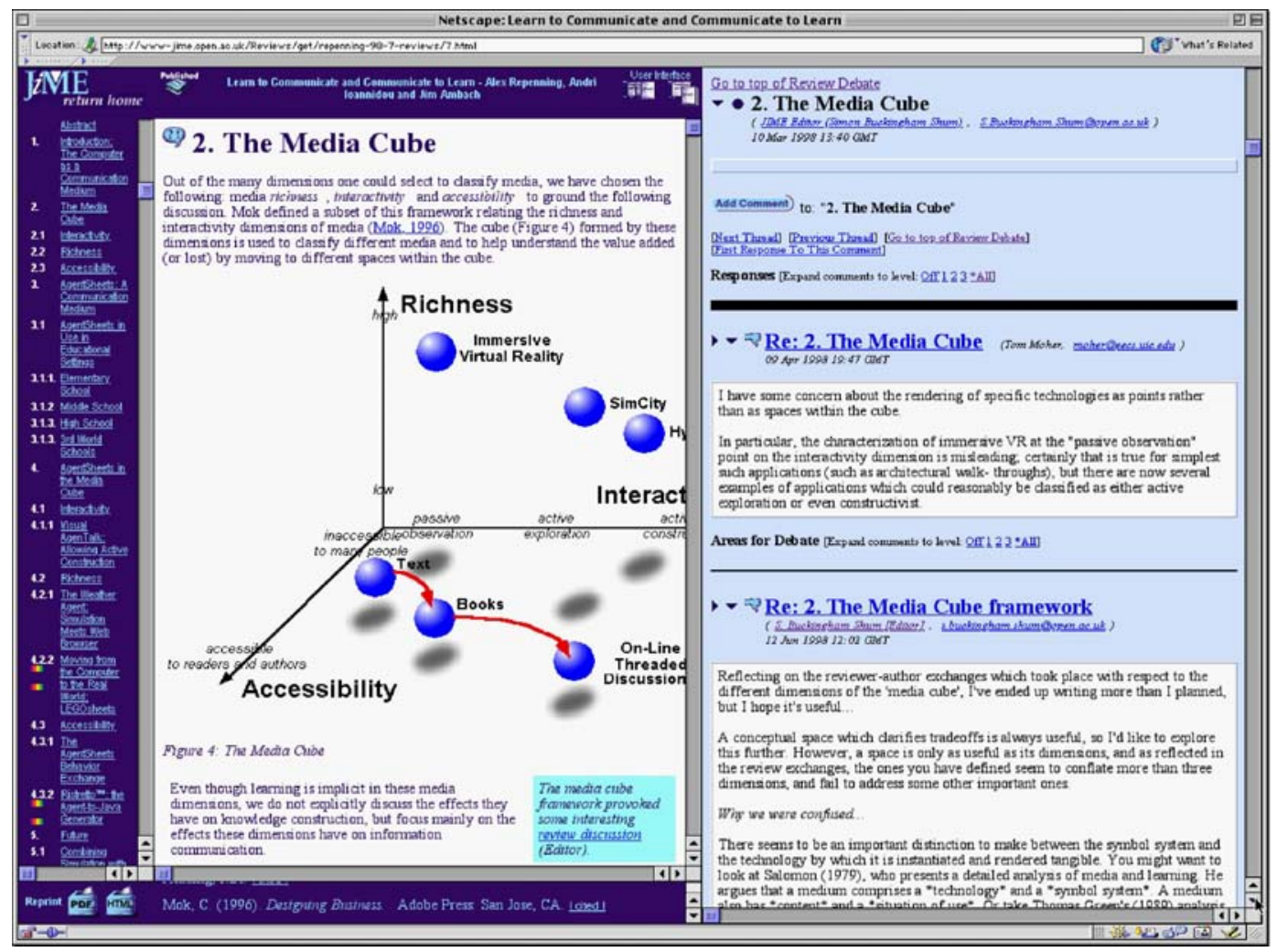

Figure 6: Article and related discussion in JiME

The peer review process of JIME is designed to promote multidisciplinary dialogue through the use of a purpose-designed Web document-discussion interface, which tightly links the article to an area for review comments and discussion (cf. Figure 6). The peer review model and the result- 
ing enriched digital documents illustrate some of the possibilities for promoting knowledge construction and preserving intellectual products in digital scholarly publications.

\section{ScholOnto}

ScholOnto is an ontology-based digital library server to support scholarly interpretation and discourse (Buckingham Shum, Motta \& Domingue, 2000). It enables researchers to describe and debate via a semantic network the contributions a document makes, and its relationship to the literature (cf. Figure 7). The system adds a semantic layer to the conventional metadata requested by providing a relatively simple set of argumentation links to make it as easy as possible to add an argumentation link to a concept or claim. The domain targeted by ScholOnto is the relatively consistent way in which researchers in a community present and contest new claims in a literature. Shifting the representational focus to the way in which researchers make new contributions to the literature avoids the problem of requiring commitment to discipline-specific ontologies that may become outdated and which may express a particular perspective. The representation of the domain in ScholOnto is only constructed in the context of authors' claims about their work, which are open to contest by others.

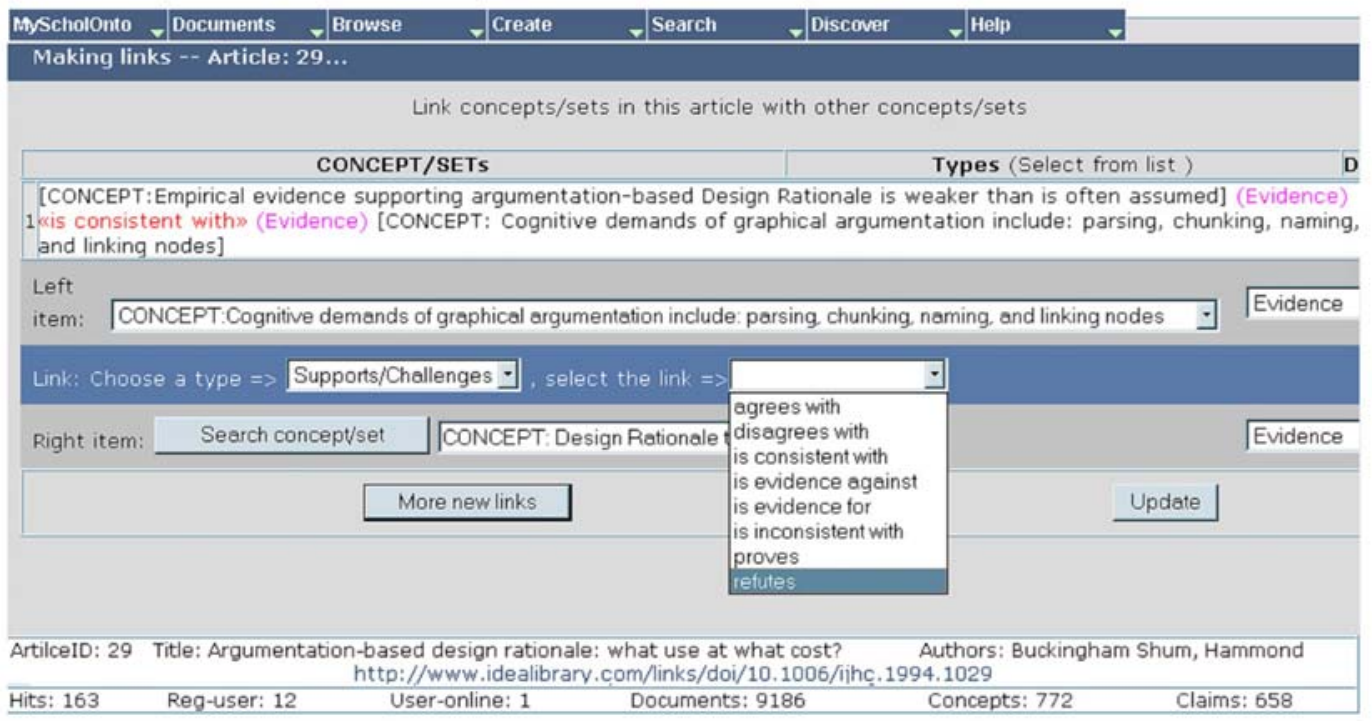

Figure 7: Linking of concepts/sets in ScholOnto

The ScholOnto server builds on a suite of robust knowledge modeling technologies developed and tested in other domains. The OCML modeling language (Motta, 1998) supports the construction of knowledge models through the specification and the operationalization of functions, relations, classes, instances and rules. It also includes mechanisms for defining ontologies and problem solving methods (Benjamins \& Fensel, 1998), the main technologies developed in knowledge modeling research. Problem solving methods are specifications of reusable problem solving behaviors.

\section{Wikipedia}

Wikipedia is an online open-content encyclopedia project, that is, a voluntary association of individuals and groups who are developing a common resource of human knowledge (cf. Figure 8). The Wikipedia concept seems quite weird at first. Wikipedia is a collaborative all in one compo- 
sition system, a discussion medium, a repository, a mail system, and a chat room. The name 'Wiki' may is strange too and comes from "wiki-wiki" which is Hawaiian for "quick" (Wagstaff, 2004). Its structure allows any individual with an Internet connection and World Wide Web browser to alter the content found here. Therefore, nothing found within the project has necessarily been reviewed by professionals who are knowledgeable in the particular areas of expertise necessary to provide a complete, accurate or reliable information about any subject in Wikipedia.

Wikipedia is a piece of server software that allows users to freely create and edit Web page content using any Web browser. It supports hyperlinks and has a simple text syntax for creating new pages and crosslinks between internal pages on the fly. Wikipedia is unusual among group communication mechanisms in that it allows the organization of contributions to be edited in addition to the content itself. Like many simple concepts, "open editing" has some profound and subtle effects on Wikipedia usage. Allowing everyday users to create and edit any page in a Web site is exciting in that it encourages democratic use of the Web and promotes content composition by non-technical users.

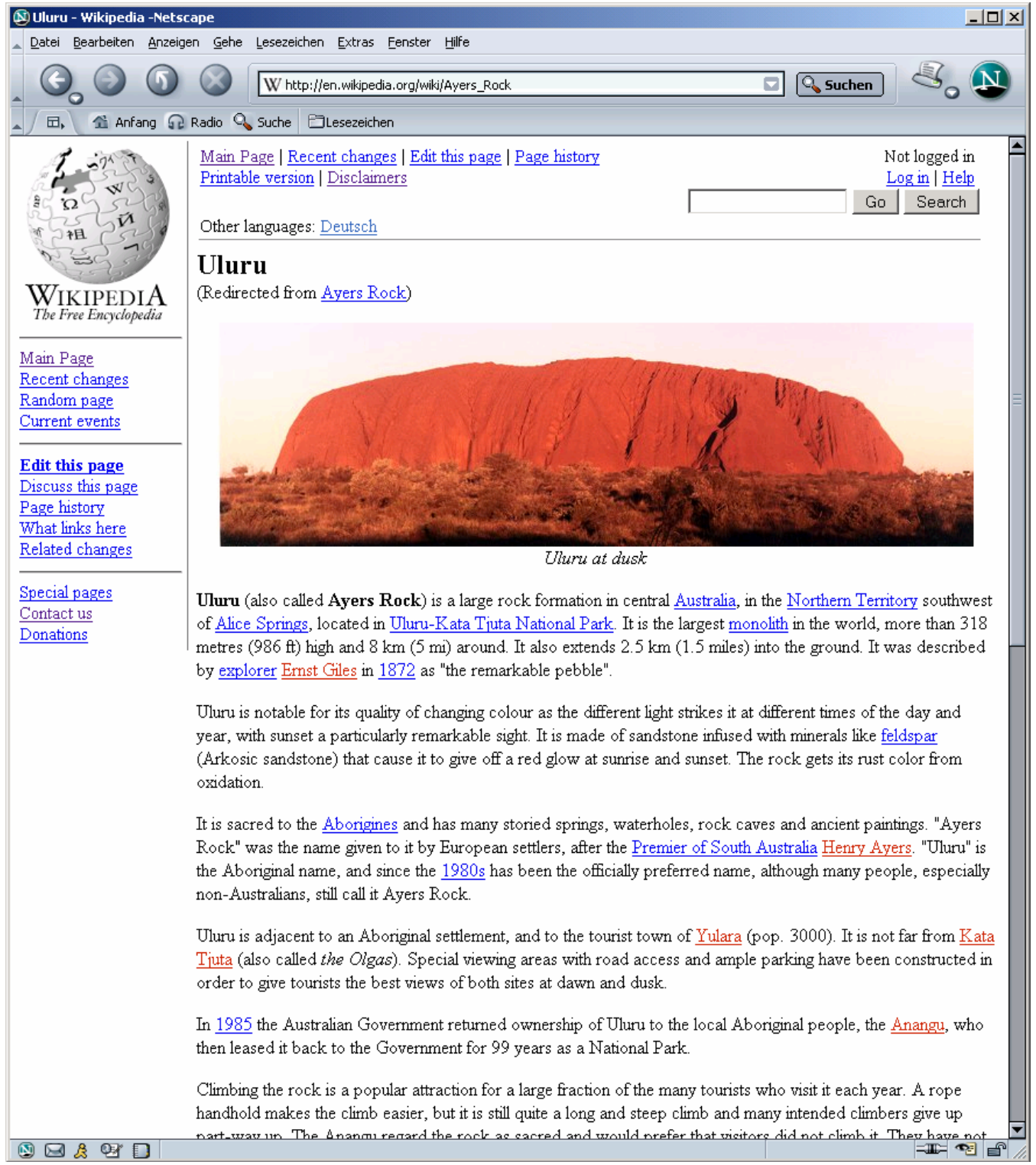

Figure 8: Wikipedia webpage on Uluru 
Recapitulating, Table 1 gives an overview on features offered by the previously introduced approaches for supporting cultural science communities. Since none of the techniques fulfills all requirements for an comprehensive support of cultural science communities we will in the following discuss repository techniques for capturing semantics of multimedia artifacts to comply with these tasks.

Table 1: Comparison of features offered by existing approaches for supporting cultural science communities

\begin{tabular}{|c|c|c|c|c|c|c|c|}
\hline & $\begin{array}{c}\text { Collective } \\
\text { multimedia } \\
\text { artifact } \\
\text { repository }\end{array}$ & Transcription & $\begin{array}{c}\text { Search } \\
\text { and } \\
\text { Retrieval }\end{array}$ & $\begin{array}{l}\text { Community } \\
\text { management }\end{array}$ & $\begin{array}{c}\text { Shareable } \\
\text { Personal } \\
\text { and Group } \\
\text { Collections }\end{array}$ & $\begin{array}{l}\text { Hypermedia } \\
\text { and } \\
\text { interrelation } \\
\text { graphs }\end{array}$ & Ontologies \\
\hline CESE & $\begin{array}{c}\text { Yes, } \\
\text { hypertexts in } \\
\text { XML format }\end{array}$ & \begin{tabular}{|} 
a-priori encoded \\
in XML; \\
a-posteriori \\
transcription not \\
supported
\end{tabular} & $\begin{array}{l}\text { Features } \\
\quad \text { for } \\
\text { searching } \\
\text { particular } \\
\text { types of } \\
\text { text }\end{array}$ & Not supported & Not available & Not supported & $\begin{array}{c}\text { Not } \\
\text { supported }\end{array}$ \\
\hline WEL & Yes & \begin{tabular}{|c|} 
a-priori \\
encoded; \\
a-posteriori \\
transcription for \\
personal use \\
possible \\
\end{tabular} & $\begin{array}{l}\text { Yes, but } \\
\text { only on the } \\
\text { original } \\
\text { material }\end{array}$ & Not supported & $\begin{array}{l}\text { Individual } \\
\text { collections } \\
\text { can be } \\
\text { checked out } \\
\text { but not } \\
\text { checked in }\end{array}$ & Not supported & $\begin{array}{l}\text { No, just } \\
\text { indexing }\end{array}$ \\
\hline $\begin{array}{c}\text { Berliner } \\
\text { sehen }\end{array}$ & $\begin{array}{l}\text { Yes, mostly } \\
\text { metadata, } \\
\text { depending on } \\
\text { the } \\
\text { application } \\
\end{array}$ & $\begin{array}{c}\text { Yes, mostly free } \\
\text { text stored in } \\
\text { Dublin Core } \\
\text { format }\end{array}$ & \begin{tabular}{|c} 
Yes, on \\
individual \\
and on \\
community \\
level \\
\end{tabular} & Yes & $\begin{array}{c}\text { Yes, } \\
\text { collections on } \\
\text { group and/or } \\
\text { community/ies } \\
\text { level } \\
\end{array}$ & $\begin{array}{l}\text { No, collections } \\
\text { only but no } \\
\text { complex } \\
\text { structures }\end{array}$ & Yes, “weak” \\
\hline $\begin{array}{c}\text { Journal of } \\
\text { Interactive } \\
\text { Media in } \\
\text { Education } \\
\left.\text { (JIME/D }{ }^{3} \mathrm{E}\right)\end{array}$ & Yes & Yes & $\begin{array}{l}\text { Only full } \\
\text { text search }\end{array}$ & Yes & Not available & Not supported & $\begin{array}{c}\text { Not } \\
\text { supported }\end{array}$ \\
\hline ScholOnto & Yes & \begin{tabular}{|c|} 
a-priori \\
encoded; \\
a-posteriori \\
transcription for \\
personal use \\
possible \\
\end{tabular} & $\begin{array}{l}\text { Yes, based } \\
\text { on the } \\
\text { ontology }\end{array}$ & Yes & Not available & Not supported & Yes, strict \\
\hline Wikipedia & Yes & $\begin{array}{c}\text { Yes, mostly free } \\
\text { text and } \\
\text { Hyperlinks } \\
\text { stored in HTML }\end{array}$ & $\begin{array}{l}\text { Only full } \\
\text { text search }\end{array}$ & Yes & Not available & $\begin{array}{c}\text { Only } \\
\text { Hyperlinks }\end{array}$ & $\begin{array}{c}\text { Not } \\
\text { supported }\end{array}$ \\
\hline
\end{tabular}

\section{Repository Techniques to Capture Semantics of Multimedia Artifacts}

New media allow cultural science communities to apply new operations such as the almost arbitrary recombination of multimedia artifacts. Digital media facilitates fast and complex structuring of information and offer them through different cultural interfaces to the scholars. However, the semantics of digital multimedia materials is very hard to capture and specific to the discourse.

Since the problem is already hard for the case of pure text, it becomes even more complicated when different media are involved. In our point of view an approach complementing existing approaches with a CoP-focused knowledge transformation process is most promising. In this aspect, 
the development of a common practice, which defines the CoP, integrates the negotiation of meaning between the participating members as well as the mutual engagement in joint enterprises and a shared repertoire of activities, symbols, and multimedia artifacts. This community practice is inseparable from issues of (individual and social) identity which is mainly determined by negotiated experience of one's self in terms of participation in a discursive community learning process concerning one's membership in a CoP.

Based on the requirements for scholarly communications by supporting collaboration and knowledge exchange in cultural science communities we've mentioned in the previous section, we'll discuss management strategies for the probably most decisive aspect for cultural science work, the multimedia artifacts. Managing of multimedia artifacts for computer scientists is at first glance somehow similar like handling any other kind of information. Therefore, we first present two strategies for the management of multimedia artifacts that have been adapted from "classical" knowledge management systems. In this aspect, we discuss multimedia file systems and ontology based information systems in more detail. Both are extremes on a scale of information management and not comparable with each other, for sure. However, we figure out that neither multimedia file systems nor ontology based information systems can cope with the requirements of cultural science communities. Finally, we introduce an approach of open multimedia archives that tries to eliminate deficits of the previously mentioned approaches and simultaneously strengthening their benefits by combining them.

\section{Multimedia file systems}

The idea of multimedia file systems is the more or less unclassified handling of multimedia artifacts on file systems. To support search and retrieval facilities it is possible to annotate those artifacts with metadata. In Microsoft Windows and Office these facilities are already integrated. In multimedia database management systems metadata support is used in an even more systematic and efficient way. Therefore, the metadata used for semantic enrichment of the original information is of greater importance than before. Metadata standardization efforts in the multimedia sector like Dublin Core and MPEG give evidence for the importance of metadata.

These metadata languages are vocabularies for describing multimedia artifacts. By allowing almost arbitrary metadata annotations on artifacts, they can be concatenated freely in particular via textual descriptions. However, a more sophisticated linkage between artifacts is not supported, restricting features for a structured content management to a minimum. For that reason, retrieval becomes more difficult and the risk of addressing inappropriate members increases, since search functionality is mostly limited to full text searches on metadata. Since communication processes among scholars within a CoP are needed, artifacts as communicative acts are stored in a common community repository to support information exchange. This process of storing depends on the policies in the CoP applied. In realistic practices, community artifacts and individual artifacts mix up (cf. Figure 9) because collaboration requires a certain amount of artifacts within a CoP. Therefore, retrieval of documents becomes more difficult and the risk of questionable selections in address space increase. 


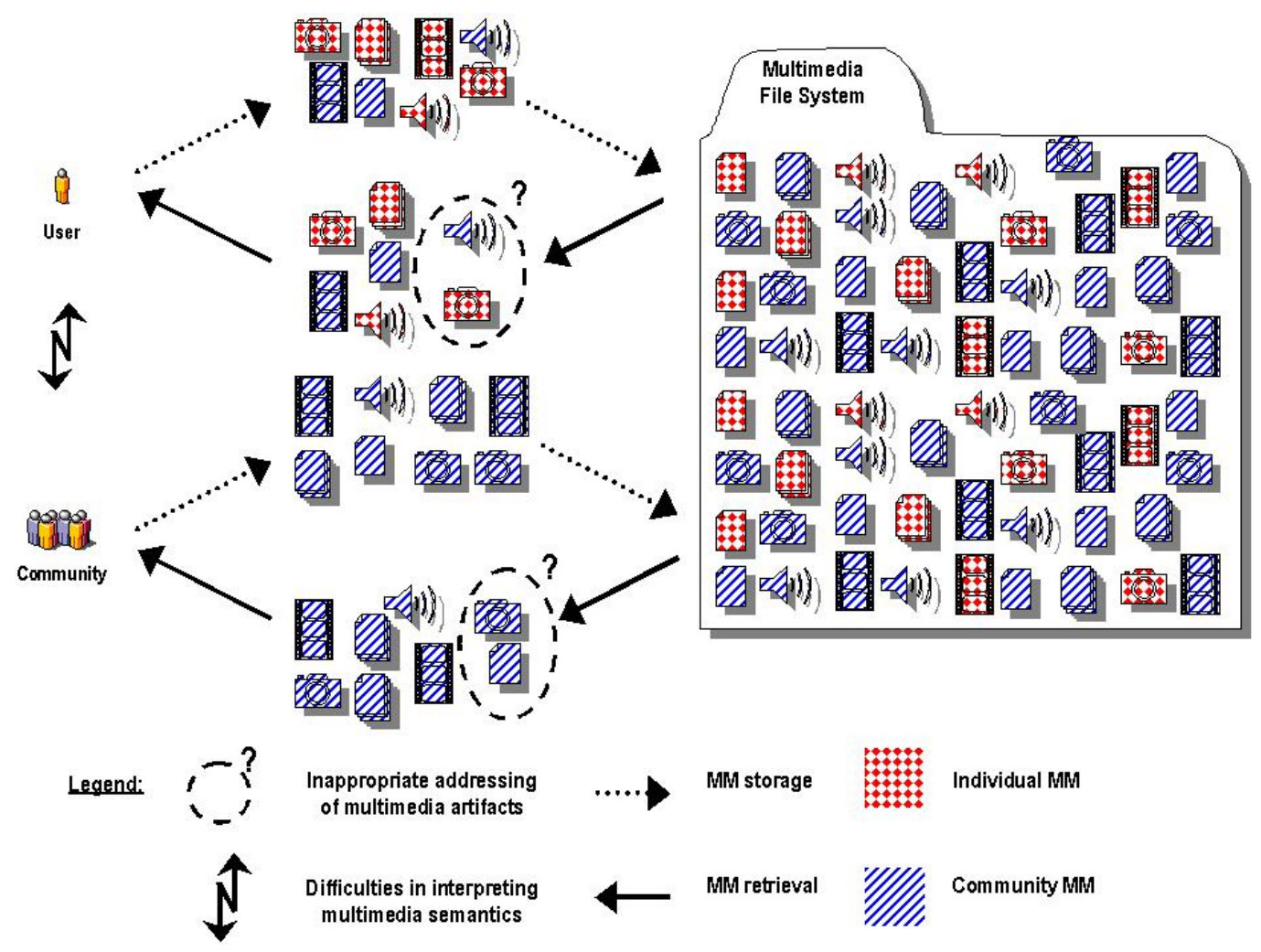

Figure 9: Managing multimedia artifacts with a multimedia file system

\section{Ontology-based information systems}

In contrast to handling multimedia on a file system, ontology-based information systems aim to structure content and support information retrieval. They range from simple catalogs to information system ontologies using full first order, higher order or modal logic (Smith \& Welty, 2001). An ontology has to fit into all user interpretations, detected during shared ontology creation. Therefore, ontology creation is usually guided by domain experts (ontology engineers) in an iterative, incremental and evaluative process. The formal languages used for evolving concepts and their interdependencies can be used for automated reasoning about dependencies between the concepts. Existing modeling techniques such as DAML+OIL (Horrocks, 2002), RDF (Reynolds, 2001), or SHOE (Heflin \& Hendler, 2000) allow an interchange of existing models among systems that understand these languages. However, an interpretation of their semantics is necessary since there doesn't exist a world formula containing all concepts and dependencies. Underlying these languages are description logics (Horrocks, 1999), FOL, or Telos (Mylopoulos, Borgida, Jarke, \& Koubarakis, 1990). Systems like ConceptBase (Jeusfeld, 1992), On-To-Knowledge (Kietz, Maedche \& Volz, 2000), or Text-To-Onto (Maedche \& Staab, 2001) allow reasoning on complex concepts or text, but neglect multimedia content. Even more, it is necessary to a-priori define a common core ontology for communication, which usually leads to an ontology creation process done by an ontology engineer acting as a mediator trying to more or less capture all relevant facts for the CoP (cf. Figure 10). Due to that, some aspects might be inside the common vocabulary confusing other users, whereas some other concepts might have been left out since they appear interesting only for a minority of users to the ontology engineer. Even if assuming high skills of the ontology engineer, the discursive nature of knowledge creation remains neglected. 


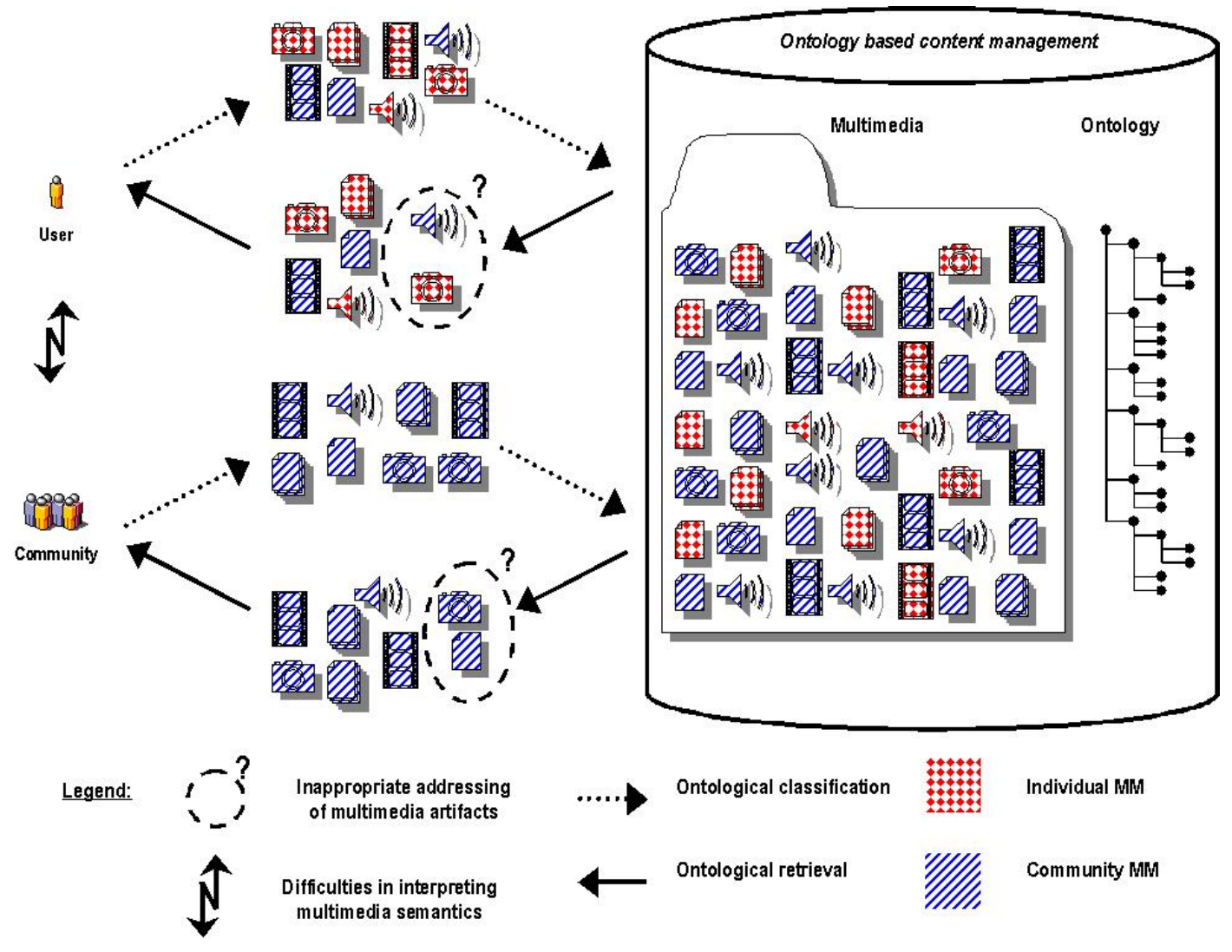

Figure 10: Ontology based management of multimedia artifacts

\section{Reflection on existing approaches}

Both, multimedia or ontology driven artifact management alone appear insufficient for hypermedia management in communities of practice. To reflect the discursive nature of communication in cultural science communities, it is necessary to integrate the vocabulary of the CoP within the language provided by an ontology as well as allowing to express almost arbitrary artifact relationships. Formal techniques can be applied to foster communication by identifying inconsistencies, redundancies, overlapping, just to mention a few. This is not to get rid of these effects but to reflect the always inherent difficulties in communication structures. Ontology based information systems allow computation of contradictions or other dependencies for a limited size of modeled concepts. Besides performance and scaling problems, ontology based information systems lack features to combine the ontologies constructed with multimedia content. Approaches can be seen in various information brokering applications but real combinational freedom for heterogeneous multimedia artifacts is still missing. Because of the inflexibility of those systems, divergent views on multimedia content for CoP and their users are hard to realize since they follow they ontology engineer model, assuming the reality for individuals as well as for CoP can be captured by his or her skills. Here, the ontology engineer is also the moderator of the communication processes within the CoP, which make his or her presence unavoidable. Those moderation processes can lead to resulting ontologies, which are common, a lowest common denominator possible, leaving many aspects unconsidered. The main advantage of artifacts managed by a multimedia file system is based on their flexibility of possible metadata annotations. However, multimedia artifacts lack modeling concepts that allow reasoning on the semantic information. Freely generated metadata information is simply almost incomparable when not standardized. Hence, reasoning on concepts and conflict detection is nearly restricted to pattern matching. Combined with the ontology 
for the CoP this situation can be improved drastically. Table 2 gives an overview on the main advantages and disadvantages of both previously mentioned techniques based on the before mentioned crucial aspects in artifact management in cultural science communities namely support of complex media interrelations, discursiveness, and openness. Obviously, neither managing artifacts via a multimedia file systems nor an ontology based information system is able to solve the problems in capturing the full richness of community oriented explicit multimedia semantics.

Table 2: Summarized reflection of multimedia artifact management via file system and ontology based information system

\begin{tabular}{|c|c|c|c|}
\hline & & $\begin{array}{l}\text { Multimedia file } \\
\text { system }\end{array}$ & $\begin{array}{c}\text { Ontology based } \\
\text { information systems }\end{array}$ \\
\hline \multirow{3}{*}{ 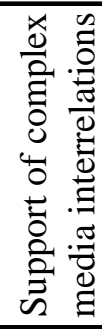 } & Modelling & weak & good \\
\hline & Reasoning & weak & good \\
\hline & Multimedia dependencies & weak & good \\
\hline \multirow{3}{*}{ 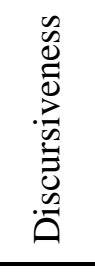 } & Distributed work & good & weak \\
\hline & Views & weak & average* \\
\hline & Individual archives & good & weak \\
\hline \multirow{2}{*}{ 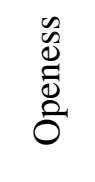 } & Content adaptability & good & weak \\
\hline & Multimedia support & good & weak \\
\hline
\end{tabular}

* good support of predifined categories

\section{Free, open, and fluid multimedia archives}

To summarize, the semantics of digital multimedia materials are very hard to capture either manually or automatically. Not only the semantics but also the interface is specific to the cultural background of the scholar. The implicit semantics of a multimedia artifact is the not necessarily shared set of interpretative acts of community members within a CoP, which emerges by discursive assignment of semantics. The explicit semantics of a multimedia artifact is the set of terms created or linked in the practice of scientific discourses, which forms the multimedia ontology of the discourse. The development of a common practice integrates the negotiation of meaning between the members as well as the mutual engagement in joint enterprises and a shared repertoire of activities, symbols, and multimedia artifacts. By focusing on discursive knowledge creation processes with semantically enriched multimedia, we extract a community terminology to categorize multimedia artifacts. By allowing scholars to modify (transcribe) different media in an arbitrary and interchangeable manner the system is capable of detecting conflicts in the explicit semantics of an individual archive and its community archive (cf. Figure 11). This keeps the dis- 
course ongoing while metadata captured by metadata standards serve as the vocabulary of transcribed multimedia artifacts.

System usability is improved by reflecting the community terminology in an underlying ontology. Deploying a standardized metadata vocabulary ensures exchangeability. The metadata description standard "Multimedia Content Description Interface” ISO/IEC 15938 1-8 (MPEG-7) ("ISO/IEC: Multimedia content description interface”, 2002) has advanced features to describe and manage multimedia artifacts as well as multimedia collections. Hence, using MPEG-7 for the capturing of semantics allows scholars to browse multimedia artifacts, multimedia collections, and hypermedia graphs. MPEG-7 features are used to capture the semantics as well as allowing users to browse by categories. In this aspect the MPEG-7 inherent graph description is used to express the semantic relation(s) between objects. We now describe the interplay of the previously mentioned aspects in our case study of the hypermedia management system MECCA.

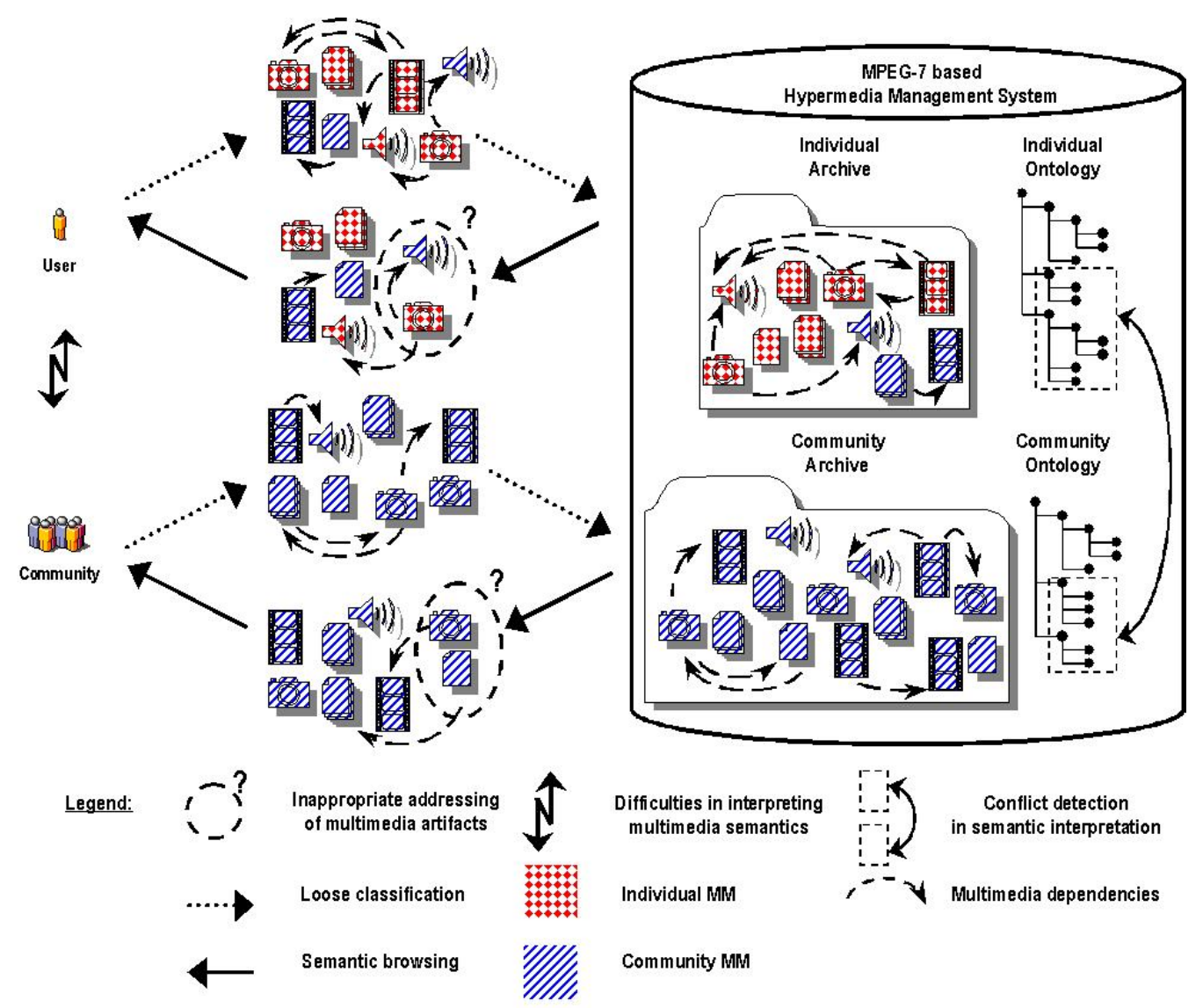

Figure 11: MPEG-7 based hypermedia management

\section{MECCA: MPEG-7 Based Support for Scholarly Dis- courses in Communities of Practice in the Humanities}

The Movie E-learning Classification and Combination Application MECCA has been specially designed for film studies communities of researchers and students within the collaborative research center "Media and Cultural Communication". Since the center covers a lot of research issues under a common "umbrella", members of the center are affiliated to CoP's inside as well as outside the center. Collaboration between different members of the center varies over time and 
new members are frequently included while others leave the center with new directions. Therefore, in the last five years some $\mathrm{CoP}$ emerged within the center. We now describe in detail the multistage community-oriented design process of MECCA and the derived requirements to serve as a multi-dimensional multimedia screening and classification platform.

\section{Open archive requirements in film studies communities}

The CoP is centered on a multidisciplinary research concerning the facial semantics in movies is cooperating with in our collaborative research center. The CoP is physically distributed in the department of film studies at the Ruhr-Universität Bochum, the center in Cologne, and Munich. Members of the CoP have diverse backgrounds of educations, e.g. film studies, history of art, graphical design and are on diverse levels of profession, i.e. full professors, research assistants, and students. The community members have already different interests and point of views, due to their educational and cultural background. Their joint enterprise is to analyze the semantics of facial expression of movies by classification of and commenting non movie scenes. They study this within four dimensions:

1) the beautiful face

2) the dramatic face

3) the portrait

4) mass faces

Three of the dimensions are assigned as a research task to a research assistant partly supported by student assistants and thesis students. The professor has the role of a research coordinator but is also responsible for the mass face dimension. Some of the students are responsible for enquiries and material collections. The size of the CoP varies. By the nature of their research tasks, the community members very often have different points of view on a shared multimedia artifact, which are expressed in a use of distinctive vocabularies. When trying to find a common level of communication the community is very often limited to restrict their vocabulary to a minimum or make use of terms that are irritating to other members having another background or research focus on the artifact. One example is the meaning of the term "text". An art historian stated that she will never use the term "text" for movies while the other members wanted to use the term in the broadest sense possible.

In the sequel, we discuss requirements for the design of the MECCA system which, for subsequent reference, are numbered R1-R6 in the text and summarized in Table 3.

A major aspect influencing the CoP is its distribution among several universities in Germany. For that reason, a collaborative computer-based system and a collective server to share multimedia files is of great importance for them (R1). Using a collective multimedia artifact server would not only foster collaboration among researchers, but also enable students to take online screenings apart from rare on-campus meetings. 


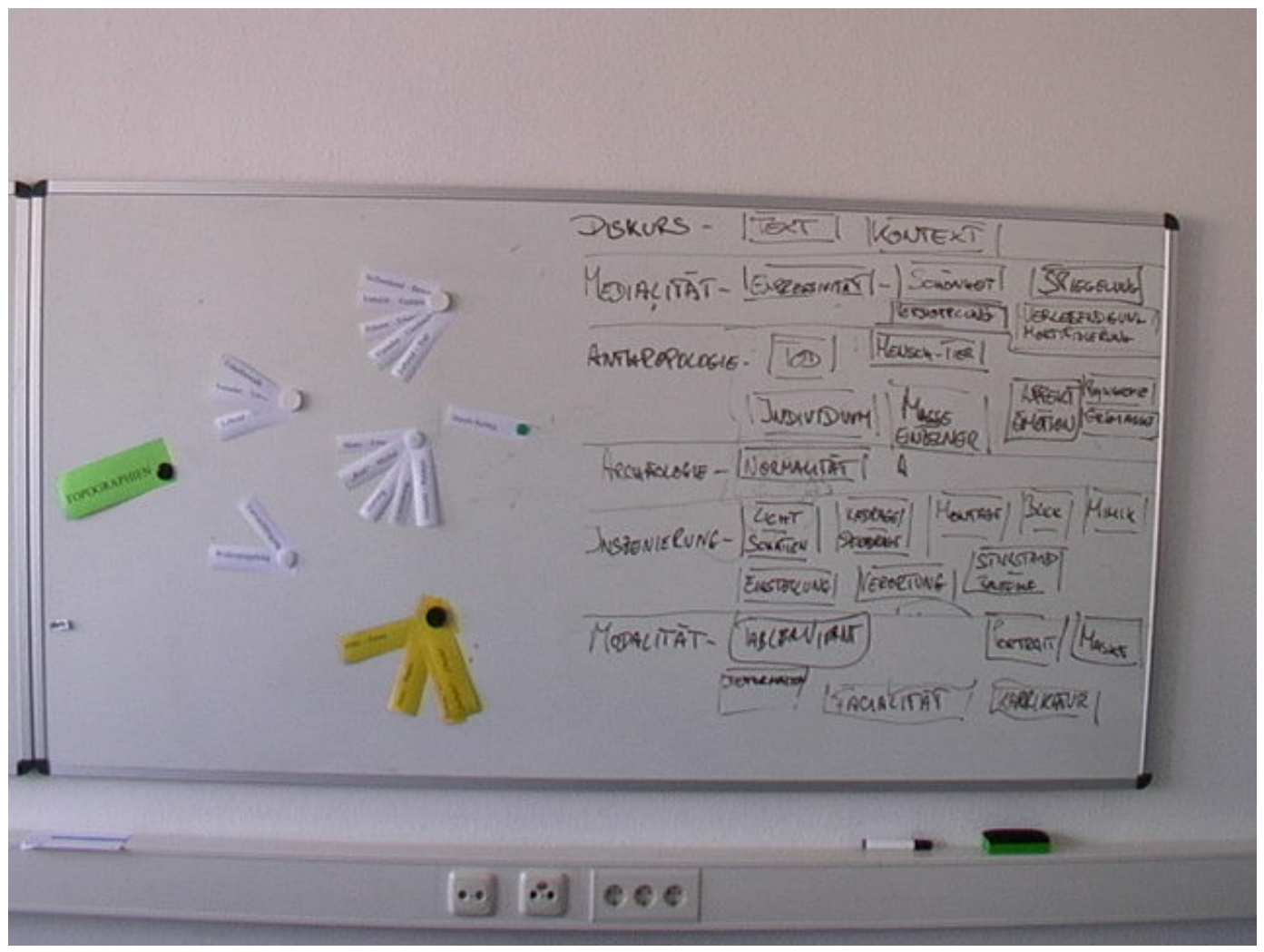

Figure 12: Categorization for movie classification to guide screenings resulting from ingroup community meetings

Then we discussed the way this CoP collaborates in case they are gathered at one place and how they learn from each other and create new knowledge. Their knowledge creation process is a discourse-oriented collaboration on the basis of movie samples covering their research topic. So, they learn by having screenings together in order to classify and comment on them. Here, we detected a crucial aspect in the CoP based on the needs for almost arbitrary classification, (re-) combination, and annotation features of multimedia artifacts to be made accessible to the community members (R2). The resulting classification scheme used for classifying movies under the focus of their research enterprise can be seen in Figure 12. It represents a semantic annotation scheme for classifying multimedia artifacts in screenings and consists of six main categories and 30 subordinate categories (now the core classification scheme has been adopted to five main categories and 28 subordinate categories) classifying a core set of 46 movie samples.

In contrast to a quite goal-oriented scholarly communication in engineering communities, we have detected a need for a more oscillating relationship of media and their underlying theory. The process is basically a continuous transcription discourse as schematically represented in Figure 2. On the one hand side, this implied an aspect of semantic freedom to be embedded into the system allowing the almost arbitrary (re-)contextualization of multimedia artifacts, but on the other hand, another requirement was derived by a demand to protect their individual property since in research communities the risk of plagiarism might not be underestimated (R3).

In addition, there is a commentary relationships among the media themselves. Information can not be regarded independent from the medium through which it is being communicated. That means, a media theory considered as an isolated information carrier of content is not sufficient, 
resulting in a need for a system carrying (at least) meta data about the situational context (background) of its creation (R4).

Nevertheless, meta data alone are unable to carry the needed information without any graphical representation. For that reason, the $\mathrm{CoP}$ needed a system allowing to express semantic relationships between various media artifacts by hiding the complexity of storing and retrieving these information. To meet the requirements of our target community as sound as possible we have searched for an alternative to let them express these relationships in an understandable and intuitive procedure similar to common working practices. Therefore, we agreed on a video editing table enriched like tool capable to visualize relationships between media (R5). Anyhow, even the commentary relationships of media didn't resolve the terminological conflicts when a common classification scheme was created.

Another system requirement of creating individual reference collections derived (branched) from the core classification scheme as it is common in paper based work in the cultural sciences. That means, community aware hypermedia requires an ability to "branch" individual knowledge creation processes for a later comparison with others or the CoP. To progress with the scientific process it is necessary not only to externalize their individual findings by a classification schema (which is just fine, e.g. for their dissertational work) but also to share and align their findings with the other members of the CoP in a common classification schema to promote the project needs (which is necessary for the research of facial semantics in movies independent from the concrete facet). Naturally, those views of individuals and the CoP are often conflicting offering innovative potential for research insights and new research questions. Instead of being restricted by a knowledge engineering system to the use of lowest common denominator ontology scientists want to discuss their different point of views and the resulting productive disturbances in the epistemological process. Furthermore, for writing research papers reflecting the discursive process of knowledge creation the CoP needs a system that allows logging and back-tracking of decisions in the common classification creation process for a better understanding of the overall process. Since, the underlying classification scheme causes such difficulties in interpreting and understanding the other members' views on a certain aspect its exchangeability and recoverability was another aspect the CoP needed to make the emerging difficulties productive for their continuous learning process (R6).

For the sake of clarity, we have summarized the previously described CoP needs in Table 3. In the following subsections we will now describe in detail how these requirements have been included in our system.

Table 3: Requirements for open archives in cultural science communities

\begin{tabular}{|c|c|}
\hline & Requirements \\
\hline R1 & $\begin{array}{l}\text { Online collaboration facilities to allow cooperative work in a } \\
\text { distributed setting }\end{array}$ \\
\hline R2 & $\begin{array}{l}\text { Arbitrary combination and (re-) classification opportunities for } \\
\text { multimedia artifacts }\end{array}$ \\
\hline $\mathbf{R 3}$ & Protecting of individual property by flexible access rights and roles \\
\hline R4 & $\begin{array}{l}\text { Information about the situational context (background) of a } \\
\text { multimedia artifact }\end{array}$ \\
\hline R5 & Presentability of complex multimedia interdependencies \\
\hline R6 & $\begin{array}{l}\text { Creation of reference collections by branching the core ontology to } \\
\text { ensure individual views }\end{array}$ \\
\hline
\end{tabular}




\section{Design and Implementation}

We now explain in depth how the required CoP tools have been integrated in our system to support cultural science work flows. We have clustered the before mentioned aspects into four subsections as they are realized in our system on a logical level and within the respective user interfaces.

\section{Collective multimedia screening environment (R1)}

In 2002, MECCA was introduced to our colleagues as a multimedia screening environment to foster scholarly collaboration in a distributed setting as well as an environment empowering students to have screenings at home instead of (or even replace) rare on-campus screenings. For that reason a prototype was built based on the Virtual Entrepreneurship Lab (VEL), which has been successfully applied in online entrepreneurship training, integrating ideas from "Berliner sehen" (Fendt, 2001).

The front end of MECCA used for screenings is show in Figure 13. The categorization schema to the right allows for metadata-mediated browsing by switching between multimedia artifacts. Keyframe thumbnails of videos vary related to the chosen category. Dragging its key-frame and dropping it in the middle play a video. Besides, all annotations concerning the media file are now accessible. By highlighting the appropriate categories depending on the context of the selected media the user is encouraged to investigate other aspects associated with the media artifact.

Scholars can collaboratively maintain collections (bottom left). Collections can be seen as an approach to focus on a certain aspect: combining and customizing media artifacts and compounding this view on single media components with additional, issue related information.

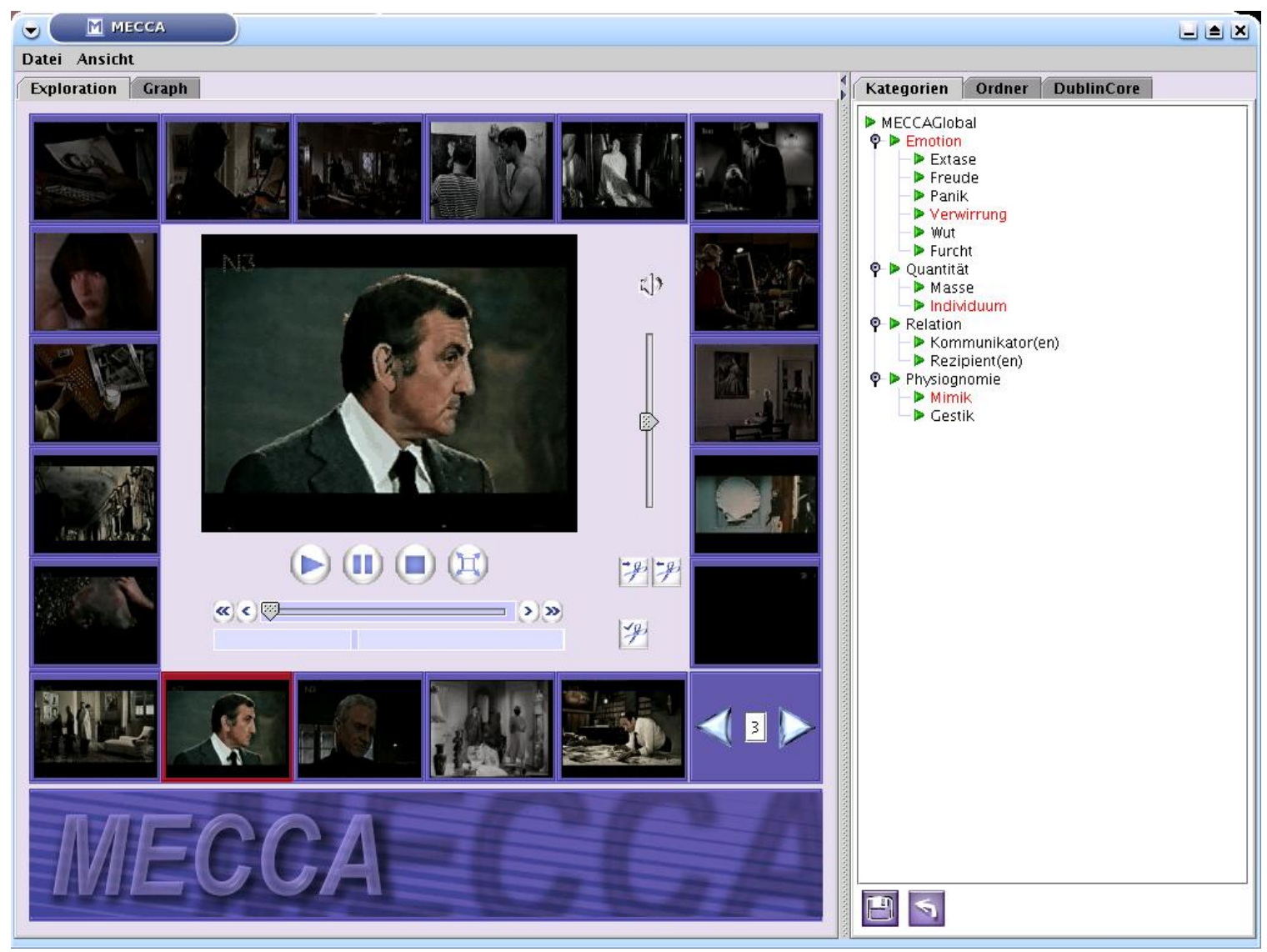

Figure 13: MECCA: Screening platform for virtual communities 


\section{Metadata management strategies for communities (R2+R3)}

As pointed out before, cultural science communities need options to annotate and (re-)classify multimedia artifacts to keep a continuous discourse going by setting media in relation to an underlying theory and reflecting the gained information on the medium again. To enable scholars to create and share almost arbitrary annotations on media files we have searched for a possibly wide spread description language. On the one hand, there exist metadata frameworks like RDF mainly originated in the environment of semantic web applications used for machine-processed description of web resources. On the other hand, standardized description schemes as Dublin Core exist for high level media descriptions. However, neither can satisfy the community's needs alone because standardized metadata frameworks tackle the problem on a very technical level, neglecting that a community of scientists in the Humanities prefers a coarser, on-the-fly classification. That is basically a feature standardized description schemes offer by excluding more sophisticated specification features apart from basic elements (e.g., there are only 15 basic element types included in Dublin Core) on their side. In this aspect, MPEG-7 closes the gap between standardized description schemes and standardized metadata frameworks. Therefore, we applied an approach based on MPEG-7 in order to offer a community specific multimedia classification, annotation, and (re-) contextualization environment.

A MECCA navigation panel allows the access of loosely classified multimedia artifacts. For this reason, the community's core ontology for browsing artifacts is stored in the MPEG-7 compliant XML database management system eXist (“eXist - Open Source Native XML Database”, 2004; Meier, 2003) connected via an Apache web server (http://www.apache.org) to enable distant collaboration (cf. Figure 11). Gradually annotating and (re-)classifying multimedia artifacts supports the discursive nature of (e-)learning in our CoP. In addition, a centralized community log-in assures that only legitimated scholars get access to the system. By doing so, we are able to protect intellectual property inherent in individual collections and annotations to be shared only among those members who might not behave selfish.

\section{Inter media relationships and context information (R4+R5)}

Since a naive media understanding is insufficient to express the full complexity inherent in media constellations present at the time point of creating collections, a tool offering to comment media on media has been integrated into our system intended to bind the situational context to the medium. The commenting tool builds on a well-known metaphor - a video editing and cutting workplace - in work processes of scientists in the CoP. MECCA's multimedia graph browser expresses hypermedia structures as shown in Figure 14. It represents the relationship between multimedia artifacts in a graphical interface based on a MPEG-7 compliant graph.

The typed relations offer an easy contextualization of hypermedia artifacts. In addition, arbitrary collections of other community members can be loaded, (re-)arranged, branched, and commented on (as long as they are public). By assigning types to the relations according to a predefined community-wide convention retrieval and comparability is increased. Furthermore, drag \& drop allows an intuitive rearrangement of hypermedia artifacts - represented by their thumbnails within the multimedia graph browser. So, it is possible at first sight to see particular media constellations of a user's collection before switching to the multimedia artifact and its (situational) annotation. To keep the discourse going on, collections within the multimedia graph browser might be freely rearranged (like the collaborations in the main frame) and commented on. An integrated tracing component allows the switch back to previous constellations and giving an opportunity to reproduce the creation and evaluation process of a particular media constellation offering an additional feature to understand the background. 


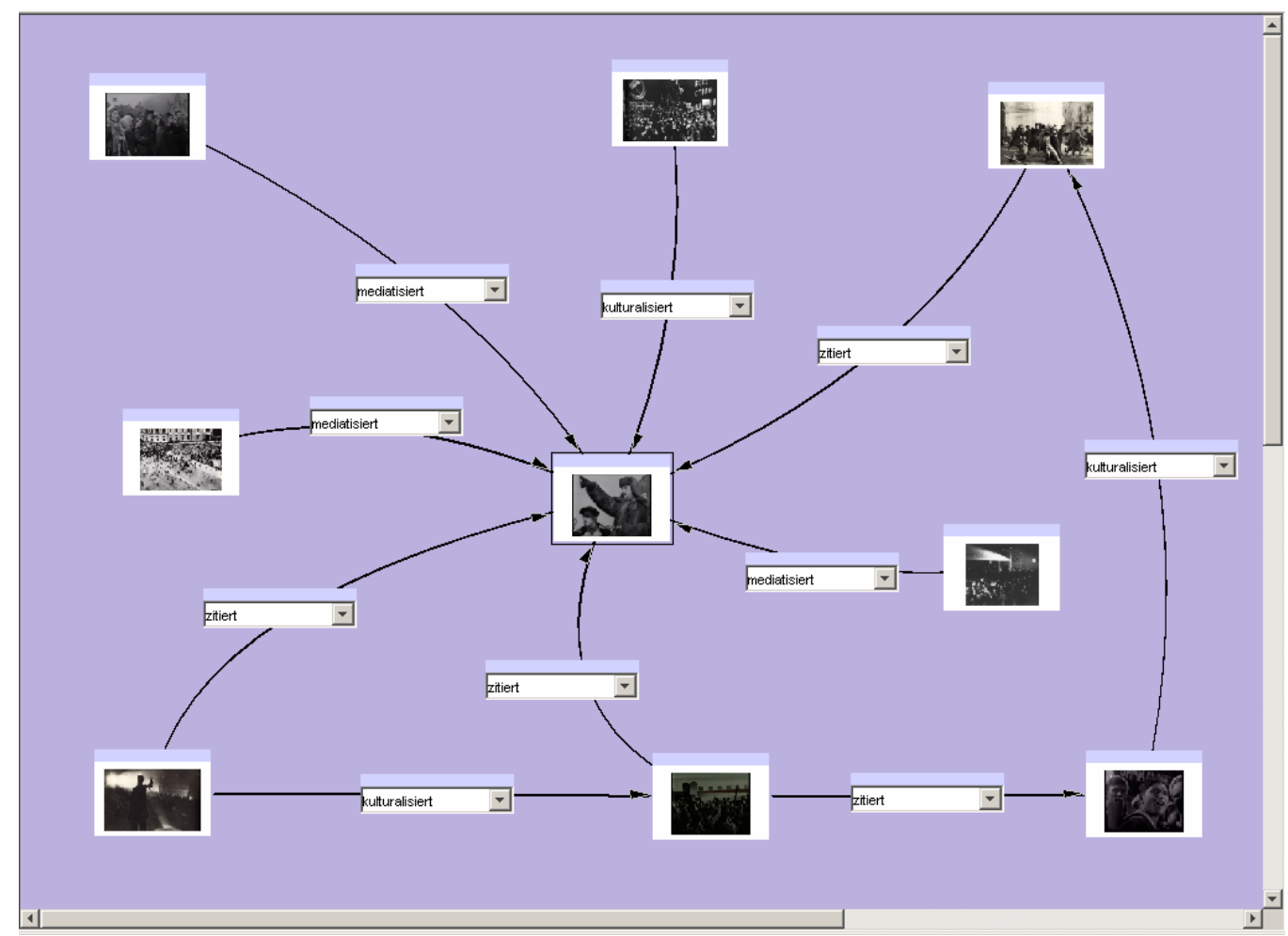

Figure 14: Media commenting on Media in the Multimedia Graph Browser of MECCA

\section{Scholarly discourses and reference collections (R6)}

A major deficit of existing systems in the cultural sciences is their restrictive administration of reference collections. In general, reference collections are possible by branching the overall system at a certain point in time. However, bringing back modifications performed individually or in collaboration on the previously checked out data set in the system is basically impossible. Some of the existing collections might be refined and rearranged almost infinitely (Fendt, 2001) but don't allow to create an individual index in terms of the categorization scheme. Other systems like the WEL (Schmidt, Sehring \& Warnke, 2001) allow to check out the overall terminology to modify the common index for individual purposes, but block its way back into the community wide established system to protect a pre-defined structure.

To allow parallel processing of hypermedia in reference collection annotations, ontologies and graph dependencies can be checked out and compared for similarities and differences. The visualization of the results is then a consequent step in supporting the discussion process to make the emerging difficulties in the semantic capturing productive. The front end of the context editor for creating fluid archives in reference collections is shown in Figure 15. It allows the individual adaptation of the underlying classification scheme as well as assigning hypermedia artifacts to certain categories. Additionally, recovery and loading features are included to exchange and discuss the categorizations within the CoP, also compared with their temporal development. 


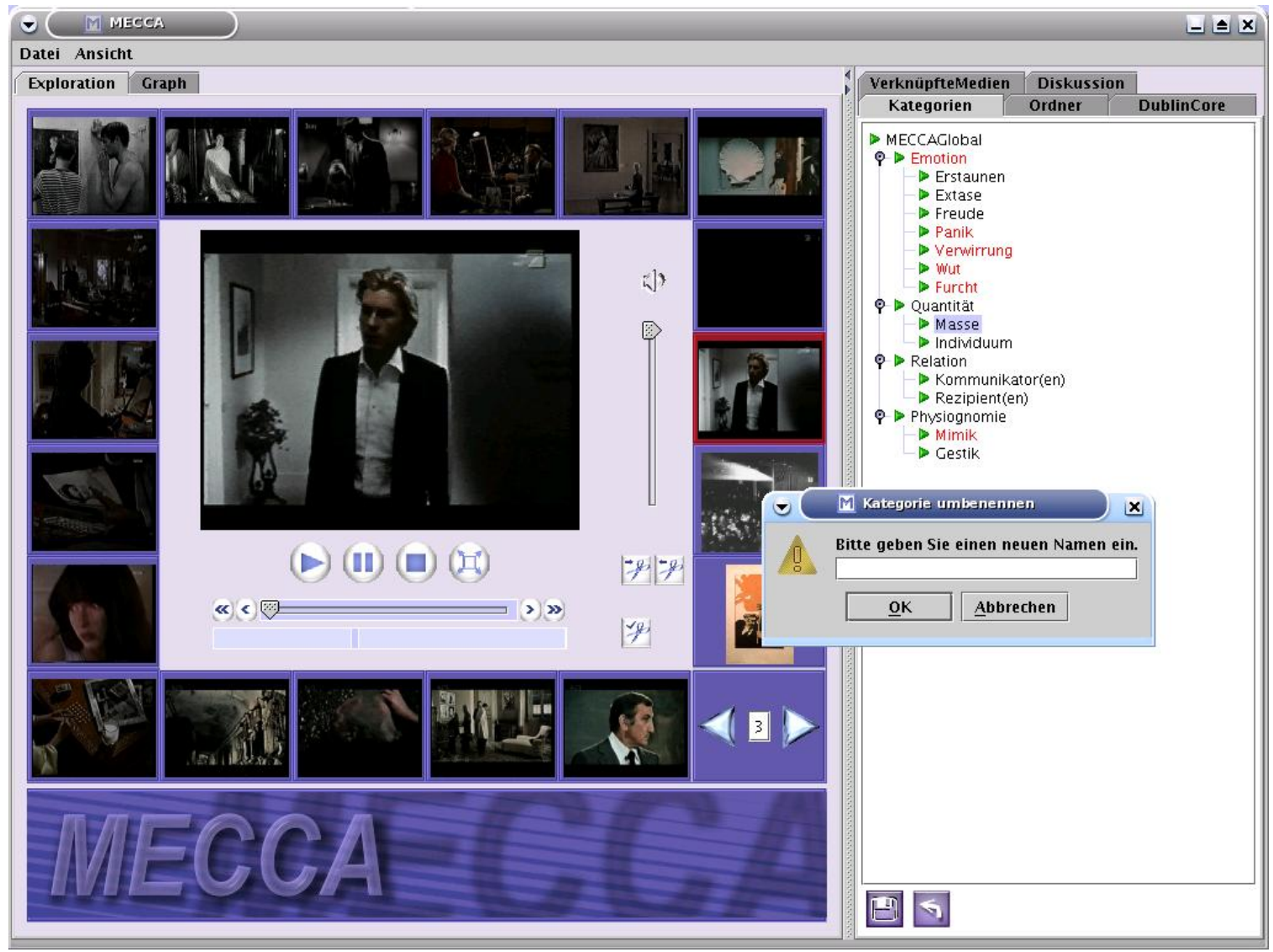

Figure 15: Creation of reference collections in MECCA by creating an individual ontology

New reference collections are created on the basis of the core ontology and the media annotations stored in the system. They are managed conjointly by dedicated (unique) name tags. We have split up these information into two documents, in order to separate changes of the meta data annotations from modifications of the ontology. Hence, meta data annotations refer to a particular tag of the XML file containing the ontology. The schema we are using to store the core ontology of MECCA as well as their individual adaptations is shown in Figure 16. The ontology itself is represented as a MPEG-7 compliant graph description. An excerpt of the core ontology is shown in Figure 17. By doing so, modifications of the ontology structure are implicitly connected to the media files related to these elements. Consequently, renaming of already existing element names can be performed without having the risk of generating inconsistencies. Creation of new categorization elements is unproblematic but due to the community's interests in expressing a hierarchy the creation of a cycle is not allowed. Deletion of ontology elements might lead to inconsistencies if media annotations would refer to that particular element. Hence, when deleting categories related media annotations are searched for references on that particular item and being deleted, in case they exist. 


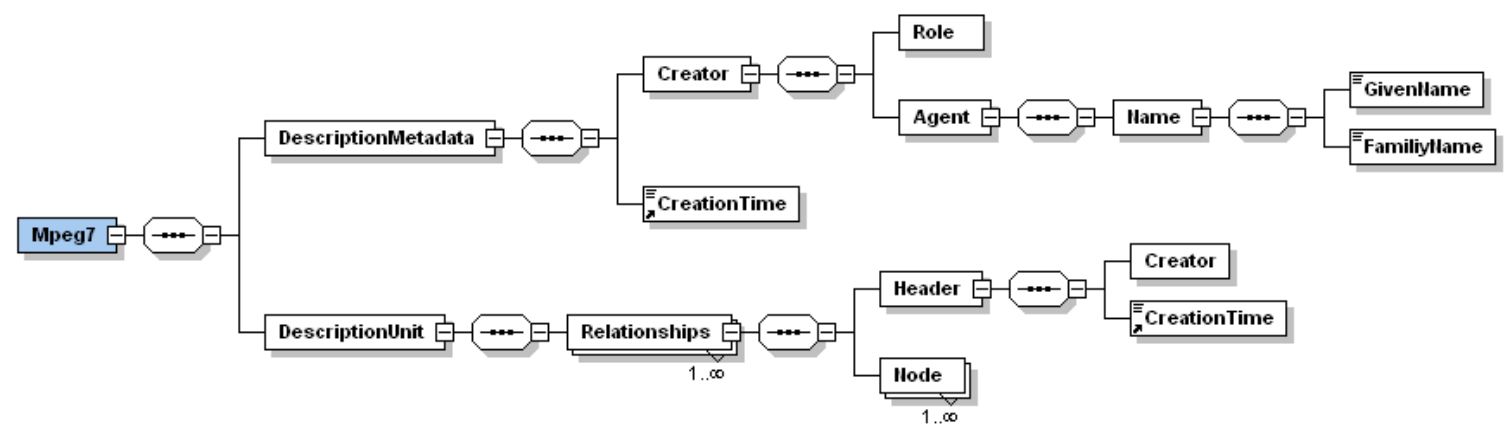

Figure 16: Categorization schema in MECCA (created with XML Spy)

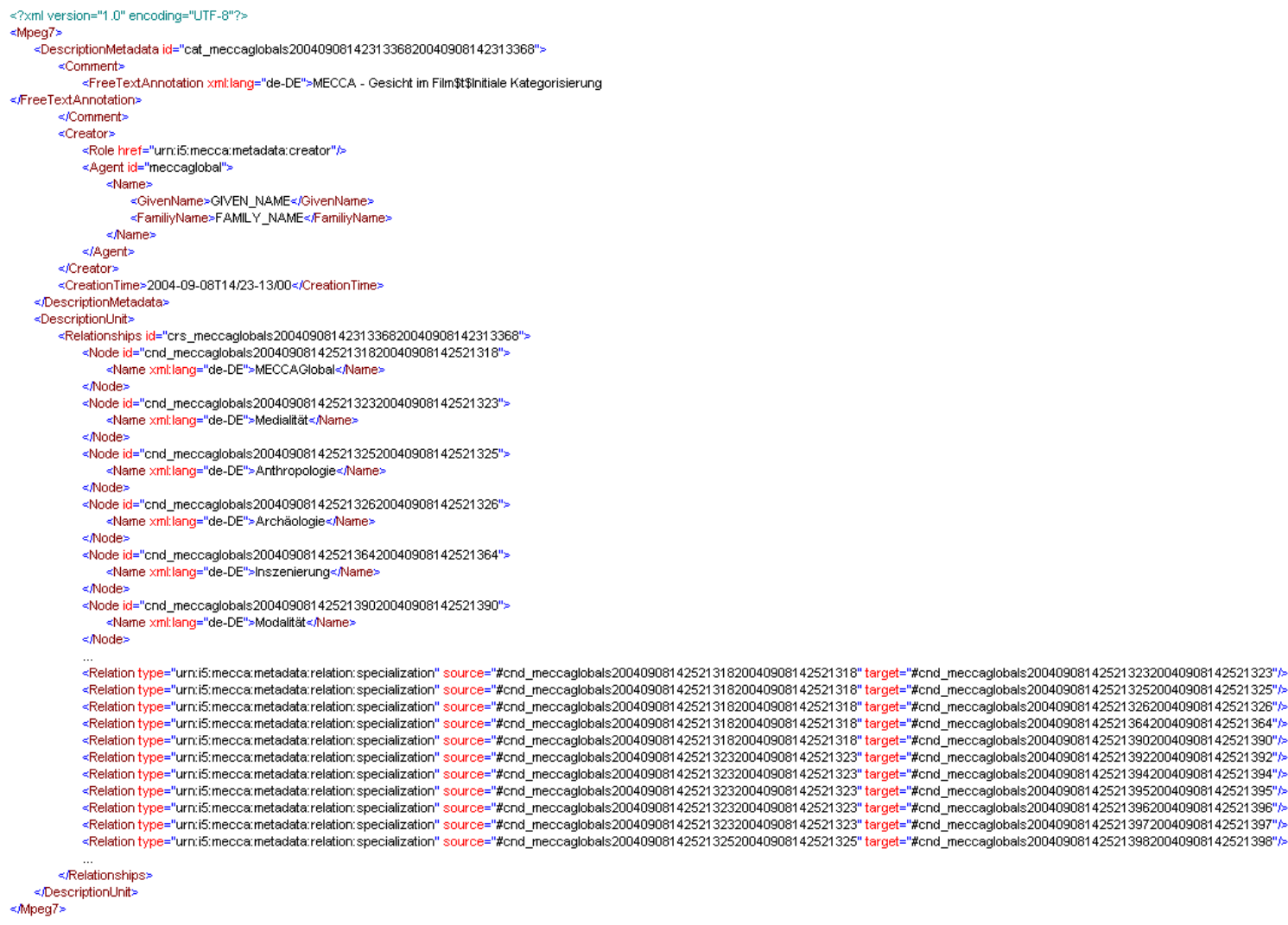

Figure 17: Excerpt of the core ontology of MECCA stored as MPEG-7 compliant graph 


\section{Ongoing Work in MECCA}

As described in the previous section MECCA offers sophisticated support for CoP in interdisciplinary projects on facial semantics in movies in a distributed setting. However, even in a CoP with a joint enterprise difficulties in interpreting and using terms arise. To detect and make these hidden conflicts productive we are currently developing tools to support a semi-automatic mediation of community ontologies for an intra- as well as an inter-community mediation system. In this aspect the COSMO system (Community Semi-automatic Matching of Ontologies) has been developed as a web service to comply with these tasks (Schmitz, 2003).

In the following, we'll describe the process of hypermedia addressing with COSMO in detail. The basis of COSMO is built on reference collections used for classifying a set of multimedia artifacts in MECCA represented as MPEG-7 compliant context graph. Since context graphs heavily depend on the user's situational background, it is very probable that these descriptions are quite different. Usually, the individual level of education and the professional background is reflected in the individual descriptions wherefore the terminology is quite different. To overcome the gap between aspects having the same semantic and being expressed unequal COSMO makes use of mediator ontologies. Mediator ontologies represent both kinds of preferences by ontologies fed by the individual terminology and an upcoming integration step by mediating concepts. To assure quality, COSMO detects concepts fully resp. partially matching. Indication is given by a probability value of concepts matching as well as marking those showing divergence with a final manual editing option for the user.

Emerging disturbances in communications are eased by the usage of implicit and explicit ontologies. The implicit ontologies are computed during runtime from the previously described MPEG-7 user descriptions. They represent the ontology inherent in a particular description and contain the terminology used in a CoP. Contrary, the explicit ontology is given by WordNet (Fellbaum, 1998), which represents a domain independent thesaurus. Both types of ontologies are managed within COSMO by the use of graph structures that are used for the upcoming computation of the semantic similarity. We combine the knowledge implicated in these ontologies when calculating the semantic similarity of concepts.

$$
\begin{aligned}
& \text { candidates }(s):=\text { candidates }_{\text {RetrieveKnownMatches }}(s) \\
& \qquad \text { candidates }_{\text {SameNameSameType }}(s) \\
& \qquad \text { candidates }_{\text {StructuralComparison }}(s) \\
& \cup \text { candidates } \\
& \text { WingleUnmatchedSibling } \\
&
\end{aligned}
$$

Figure 18: Heuristics applied in COSMO

COSMO is a fix point based approach that makes use of four heuristics that are applied step by step to find the set of potential candidates being similar in source and target ontology represented 
by a mediator ontology (cf. Figure 18). The computation results in a (if there are any matching candidates only) set of potential matching candidates denominated with a matching probability. Per iteration the algorithm seeks for a concept of the source ontology a set of potential matching candidates originated in the target ontology involving natural language aspects like polysems, holonyms, and hypernyms as well as lexical, structural, and thesaurus dependencies. Those concepts are pruned directly that have a lower probability in being a matching candidate than an apriori defined threshold value requires increasing the efficiency of our algorithm. Hence, we are able to mediate conceptualizations within or among communities.

\section{Lessons Learned}

Based on the positive comments by the CoP itself, and scholars' incitations on creating fluid archives, we have achieved designing the MECCA system, allowing individual screenings guided by a loose classification schema. MECCA aims at an interdisciplinary community of researchers interested in studying the facial semantics within movies. The system has been initially introduced to the researchers and students of the film studies especially for the usage in screenings. Due to discursive knowledge creation processes in the CoP there is a strong interest in exploring distributed classification processes of individuals or CoP in contrast to centralized ones. Logging and tracing of decisions are now recoverable and we are planning to integrate visualization facilities in the context of artifact clustering. By using these techniques we hope to detect a model core common in all community-oriented learning and knowledge creation processes.

The use of MPEG-7 has been crucial for the development of MECCA which reflects the state of the art in the didactics and interdisciplinary research within the department of film studies. We are able to express multimedia semantics by annotating multimedia in a collaborative process of knowledge creation. Furthermore, we are able to contextualize heterogeneous information since MPEG-7 allows us to import and process versatile information from arbitrary digital media formats.

Table 4: Matching requirements in cultural science communities with case study results

\begin{tabular}{|c|c|c|c|c|c|c|c|}
\hline & $\begin{array}{c}\text { Collective } \\
\text { multimedia } \\
\text { artifact } \\
\text { repository }\end{array}$ & Transcription & $\begin{array}{c}\text { Search } \\
\text { and } \\
\text { Retrieval }\end{array}$ & $\begin{array}{c}\text { Community } \\
\text { management }\end{array}$ & $\begin{array}{c}\text { Shareable } \\
\text { Personal } \\
\text { and Group } \\
\text { Collections }\end{array}$ & $\begin{array}{c}\text { Hypermedia } \\
\text { and } \\
\text { interrelation } \\
\text { graphs }\end{array}$ & Ontologies \\
\hline R1 & X & X & & X & X & & \\
\hline R2 & X & X & X & & X & & \\
\hline R3 & X & & X & X & X & & \\
\hline R4 & X & X & & & & X & \\
\hline R5 & X & & X & & & X & \\
\hline R6 & X & & & X & X & & X \\
\hline
\end{tabular}

Legend: $\mathrm{X}$ indicates required community tools in cultural science discourses

We will now discuss in how far the requirements extracted from the analysis of our target CoP with the requirements for the support of communities in the humanities in general have been met against the background of our case study. The results of this comparison can be found in Table 4 .

As we can conclude from the results in the table, the focal point for collaboration in cultural science communities is a collective multimedia artifact repository since it represents the basis for all activities within the CoP. This requirement makes a logical united repository essential. However, this might lead to problems in case of CoP having only part time access to web resources. Hence, synchronization features might be useful to offer an offline work modus in future. 
The transcription issues are needed not only explain the artifacts, but also to explain their situational background, and to offer commentary functionality, in general. Since the transcription is an essential element in making documents more readable it is indispensable to support discourses in cultural science communities.

In a similar sense personal and group collections are used but here the need for protecting intellectual property and branching collections in reference collections, too. Of course, CoP are constituted by persons sharing a common value. However, there are members in a CoP that might not be involved in any discussion as it is with the writing of research papers by involving researchers but ignoring students (in general). That means collections exist not only on community level but among and between sub-communities, too.

Consequently, this leads to a more or less fine grained community self-organization issue particularly to protect the intellectual property of individuals. This is disturbing the theory of CoP but inevitable because researchers have to follow their own career plans besides of shared practice. To assure that, again a centralized component - as it is on a different level with the collective repository, too - is needed to identify a CoP as itself. That means a suchlike module performs localization to assign the designated roles to a community level. Consequently, based on the role of a community member within the system the person might be allowed to perform only a limited set of actions or might not be allowed to access all the documents stored within collections.

Another aspect are search and retrieval features to find again the hypermedia artifacts and comments on them not only on the global hypermedia artifact set, but also on shareable group collections, too. What we have learned from the discussions with our colleagues that discourses in the humanities commonly start (similar at it is in engineering) with a research on results achieved in former discourses. In contrast to approaches in engineering, the outcome of the newly initiated discourse is not yet determined by a predefined goal to achieve. Following Focault, we can say that the discourse creates the things it is talking about. Even more, the researchers aim at refining the underlying theory to be applied on hypermedia artifacts as a result of their discourse. Therefore, the retrieval and re-contextualization of previous discussion stored in collections and hypermedia structures is of even greater importance than before.

Consequently, the aspects of hypermedia relationships to be stored in designated graphs is a result of the needs in capturing the situational background of an artifact as well as its dependencies with others, e.g. to express whether it is a commentary or not, seems to be appropriate to comply with this task. Anyhow, there is currently still a debate going on in how fare hypermedia and interrelation graphs might be able to capture the full expressiveness of complex media dependencies.

Last but not least, ontologies have to be considered when the core categorization is being branched in order to create reference collections of individuals. This seems to be one of the most advanced and innovative features in MECCA and is highly desired by our target CoP. The reason therefore is, that the creation of reference collections is a common working practice of studies in the humanities but has not yet been integrated in computer based applications.

\section{Conclusions \& Outlook}

We presented and discussed an information system to support scientific discourses in crossdisciplinary CoP. In our MECCA case study we showed that the use of multimedia description standards support open, scalable and fluid repositories for capturing, visualizing, and supporting scientific discourses. Standards like MPEG-7 provide a common ontology language both for the multimedia artifacts and the community vocabulary, making collaboration more transparent and flexible. In using rich media annotations and knowledge engineering methods interrelated, results in a knowledge creation process are more authentic, transparent, and flexible. Even more, the ma- 
turity of systems supporting discourses in the CoP increases, by making information systems compatible to the MPEG-7 standard.

The production and maintenance of information systems supporting collaboration in cultural science communities is still in is infancy compared to commercial ERP and CASE tools. But, insights gained from the analysis and support of scientific communities can be transferred in nextgeneration business and engineering information systems, as knowledge management using rich media and informal structures are complementing the traditional structured tools. In this sense, the case study is a promising basis to construct a meta design environment for cooperative knowledge management systems also in business and engineering settings.

Further research aims at integrating social network analysis (Degenne \& Forsé, 1991; Scott, 2000) methods into our system for systematizing knowledge about actors in networked information systems. For now, we know that scholars have relations among each other different in number and quality. By identifying the actors position in the network support can even be more specific for scholars. We are currently working together with the institute for the history of urban planning at the RWTH Aachen on a project for the re-building of scientific structures in Afghanistan. A collaborative hypermedia repository with information about historic sites and monuments aims to help the scattered community of scientists related to preservation of cultural heritage to share knowledge and to build new scientific relations, especially between the pre-war and postwar generation of scientists.

\section{Acknowledgements}

This work was supported by German National Science Foundation (DFG) within the collaborative research center SFB/FK 427 "Media and cultural communication", and by the $6^{\text {th }}$ Framework IST programme of the EC through the Network of Excellence in Professional Learning (PROLEARN) IST-2003-507310. We'd like to thank our colleagues for the inspiring discussions.

\section{References}

Ackerman, M. S. \& McDonald, D. W. (1996). AnswerGarden2: Merging organizational memory with collaborative help. Proceedings ACM Conference on Computer-Supported Cooperative Work, 97-105. New York: ACM Press.

van Beek, P., Yoon, K. \& Ferman, A. M. (2002). User interaction. In B. S. Manjunath, P. Salembier, \& T. Sikora (Eds.), Introduction to MPEG-7 - Multimedia content description interface (pp. 163-175). John Wiley \& Sons.

Benjamins, V. R. \& Fensel, D. (Eds.) (1998). Special issue on problem solving methods. International Journal of Human-Computer Studies, 49, 4.

Berners-Lee, T. (2003). Fitting it all together. Keynote Talk at WWW 2003, Budapest, Hungary.

Berners-Lee, T., Hendler, J. \& Lassila, O. (2001).The semantic web. Scientific American, May.

Bray, T. (1998). RDF and metadata. Retrieved from http://www.xml.com/pub/a/98/06/rdf.html

Brown, A. L., Ash, D., Rutherford, M., Nakagawa, K., Gordon A. \& Campione, J. C. (1993). Distributed expertise in the classroom. In G. Salomon (Ed.), Distributed cognitions - Psychological and educational considerations (pp. 188-228). Cambridge University Press.

Bruckman, A. (1994). Programming for fun: MUDs as a context for collaborative learning. Proceedings of the National Educational Computing Conference, Boston, USA.

Bruckman, A. (1998). Community support for constructionist learning. Computer Supported Cooperative Work, 7 (1/2), 47-86. 
Brusilovsky, P. (1996). Methods and techniques for adaptive hypermedia. User Modeling and UserAdapted Interaction, 6 (2/3), 87-129.

Buckingham Shum, S., Blandford, A., Duke, D. J., Good, J., May, J., Paternò, F. \& Young, R. (1996). Multidisciplinary modelling for user-centred system design: An air-traffic control case study. BCS HCI, 201-219.

Buckingham Shum, S., Motta, E., Domingue, J. (2000). ScholOnto: An ontology-based digital library server for research documents and discourse. International Journal on Digital Libraries, 3 (3), August/September. Springer-Verlag.

Buckingham Shum, S. \& Sumner, T. (2001). JIME: An interactive journal for interactive media. Technical Report KMI-TR-99. Knowledge Media Institute, Open University, UK.

Bush, V. (1945). As we may think. Atlantic Monthly, 176 (1), 101-108.

Carrer, M., Ligresti, L., Ahanger, G. \& Little, T. D. C. (1997). An annotation engine for supporting video database population. Multimedia Tools and Applications, 5 (3), 233-258.

Collins, A., Brown, J. S. \& Newman, S.E. (1989). Cognitive apprenticeship: Teaching the crafts of reading, writing and mathematics. In L. B. Resnick (Ed.), Knowing, Learning, and Instruction (pp. 453-494). Hillsdale: Lawrence Erlbaum Associates.

Conklin, J. (1987). Hypertext: An introduction and survey. IEEE Computer, 9 (20), 17-41.

Daft, R. L. \& Lengel, R. H. (1986). Organizational information requirements, media richness and structural design. Management Science, 32 (5), 554-571.

Degenne, A. \& Forsé, M. (1991). Introducing social networks. London: Sage Publication.

Dhraief, H., Nejdl, W., Wolf, B. \& Wolpers, M. (2001). Open learning repositories and metadata modeling. Proceedings of the 1st Semantic Web Working Symposium, Stanford, 495-514.

Doerr, M., Hunter, J. \& Lagoze, C. (2003). Towards a core ontology for information integration. Journal of Digital Information, 4 (1). Retrieved from http://jodi.ecs.soton.ac.uk/Articles/v04/i01/Doerr/

Dönderler, M. E., Saykol, E., Ulusoy, Ö., \& Güdükbay, U. (2003). BilVideo: A video database management system. IEEE Multimedia, Multimedia-At-Work, 10 (1), 66-70.

Duffy, T. M. \& Jonassen, D. H. (Eds.) (1992). Constructivism and the technology of instruction: A conversation. Hillsdale: Lawrence Erlbaum Associates.

Dumas, M., Lozano, R., Fauvet, M.-C., Martin, H. \& Scholl, P.-C. (2000). Orthogonally modeling video structuration and annotation: Exploiting the concept of granularity. Proceedings of the AAAI Workshop of Spatial and Temporal Granularity, AAAI Press, Menlo Park, USA, 37-43.

eXist - Open source native XML database. (2004). Retrieved from http://exist.sourceforge.net/

Fendt, K. (2001). Contextualizing content. In M. Knecht \& K. von Hammerstein. (Eds.), Languages across the curriculum (pp. 201-223). Columbus, OH: National East Asian Language Resource Center.

Fellbaum, C. (1998). WordNet: An electronic lexical database. Cambridge, MA: MIT Press.

Foucault, M. (1982). Archeology of knowledge. Pantheon Books.

Garzotto, F., Mainetti, L. \& Paolini, P. (1995). Hypermedia design, analysis, and evaluation issues. Communications of the ACM, 38 (8), 74-87.

von Glasersfeld, E. (1996). Radikaler konstruktivismus, ideen, ergebnisse, probleme. Suhrkamp, Frankfurt am Main.

Gomez-Peres, A., \& Corcho, O. (2002). Ontology languages for the semantic web. IEEE Intelligent Systems 17 (1), 54-60. 
Golshani, F., \& Vissicaro, P. (2002). Design of a multi-ethnic dance information repository. In B. Grosky (Ed.), Proceedings of SOFSEM 2002 Workshop on Multimedia Semantics, Milovy, Czech Republic, November, 27-28.

Grosky, W. I., \& Sreenath, D. V. (2001). Metadata-mediated browsing and retrieval in semantically-rich cultural image collections. In Proceedings of the 2001 Tokyo Symposium for Digital Silk Roads, Tokyo, Japan.

Gruber, T. R. (1993). A translation approach to portable ontologies. Knowledge Acquisition, 5 (2), 199-220.

Guarino, N. (1998). Formal ontology and information systems. Proceedings of the 1st International Conference on Formal Ontologies in Information Systems, FOIS'98, Trento, Italy, 3-15.

Heflin J. \& Hendler J. (2000). Dynamic ontologies on the web. Proceedings of the Seventeenth National Conference on Artificial Intelligence (AAAI-2000). AAAI/MIT Press, Menlo Park, CA, pp. 443-449.

Henze, N. (2000). Adaptive hyperbooks: Adaptation for project-based learning resources. Ph.D. Thesis, University of Hanover.

Hillmann, D. (2001). Using Dublin Core, Dublin Core metadata initiative recommendation. Retrieved from http://dublincore.org/documents/2001/04/12/usageguide

Hollender, E., Klamma, R., Börner-Klein, D. \& Jarke, M. (2001). A comprehensive electronic study environment for a Talmudic tractate. Dutch Studies - of the Near Eastern Languages and Literatures (DSNELL), IV (2), 219-245.

Horrocks, I. (1999). FaCT and iFaCT. Proceedings of the International Workshop on Description Logics (DL'99), Linköping, Sweden, CEUR-WS 22, 133-135.

Horrocks, I. (2002). DAML+OIL: A reasonable web ontology language. In Jensen et al. (Eds.), EDBT 2002: Conference on Extending Database Technology. Springer Verlag, LNCS 2287, 2 -13.

Hunter, J. (2001a). Adding multimedia to the semantic web - Building a MPEG-7 ontology. Proceedings of the First Sematinc Web Working Symposium (SWWS), 261-281.

Hunter, J. (2001b). MetaNet - A metadata term thesaurus to enable semantic interoperability between metadata domains. Journal of Digital Information, 1 (8).

ISO/IEC: Information technology - Multimedia content description interface - Part 8: Extraction and use of MPEG-7 descriptions. (2002). International Organization for Standardization.

Jäger, L. (2002). Transkriptivität. Zur medialen logik der kulturellen semantik. In L. Jäger \& G. Stanitzek (Eds.), Transkribieren - Medien/Lektüre (pp. 19-41). Munich: Wilhelm Fink Verlag, (in German).

Jäger, L. \& Stanitzek, G. (Eds.) (2002). Transkribieren - Medien/Lektüre. Munich: Wilhelm Fink Verlag, (in German).

Jarke, M., Klamma, R. \& Lyytinen, K. (2005). Meta modeling. Cambridge: MIT Press, (in print).

Jeusfeld, M. (1992). Änderungskontrolle in deduktiven Objektbanken. In DISKI 19, infix Publ. Bad Honnef, Germany. Dissertation, University of Passau, (in German).

Jonassen, D. H. \& Mandl, H. (Eds). (1990.) Designing hypermedia for learning. Part 1: Hypermedia and learning. Berlin: Springer.

Jonassen, D. H., Dyer, D., Peters, K., Robinson, T., Harvey, D., King, M. \& Loughner, P. (1997). Cognitive flexible hypertext on the web: Engaging learners in meaning making. In B. H. Khan (Ed.), Webbased Instruction. Englewood Cliffs.

Kaplan, S. M, Tolone, W. J., Carroll, A. M., Bogia, D. P. \& Bignoli, C. (1992). Supporting collaborative software development with ConversationBuilder. Proceedings of the 5th ACM SIGSOFT Symposium on Software Development Environments, ACM Press, 11-20. 
MECCA: Hypermedia Capturing of Collaborative Scientific Discourses about Movies

Klamma, R., Hollender, E., Jarke, M., Moog, P., \& Wulf, V. (2002). Vigils in a wilderness of knowledge: Metadata in learning environments. Proceedings of IEEE International Conference on Advanced Learning Technologies (ICALT 2002), Kazan, Russia, September 9-12, 2002, IEEE Learning Technology Task Force, 2002, 519-524.

Kietz, J.-U., Maedche, A. \& Volz, R. (2000). A method for semi-automatic ontology acquisition from a corporate intranet. In Aussenac-Gilles, Biebow, \& Szulman (Eds.), Proceedings of the EKAW'2000 Workshop on Ontologies and Texts.

Kosch, H. (2002). MPEG-7 and multimedia database systems. SIGMOD Records, ACM Press, 31, June, 34-39.

Lave, J. \& Wenger, E. (1991). Situated learning: Legitimate peripheral participation. Cambridge University Press.

Lemke, J. L. (2002). Travels in hypermodality. Visual Communication, 1(3), 299-325.

Lennon, J. \& Maurer, H. A. (1996). Aspects of large World Wide Web systems. Proceedings of WebNet 96 - World Conference of the Web Society San Francisco, California, USA, October 15-19, AACE.

Ligorio, M. B. (2001). Euroland: A virtual community. Proceedings of the European Conference on Computer Supported Collaborative Learning, Maastricht, The Netherlands, 731.

Looi, C.-K., \& Ang, D. (2000). A multimedia-enhanced collaborative learning environment. Journal of Computer Assisted Learning, 16 (1), 2-13.

Maedche, A. \& Staab, S. (2001). Learning ontologies for the semantic Web. In Decker, \& D. Fensel (Eds.), Proceedings of the Second International Workshop on the Semantic Web - SemWeb 2001.

Manovich, L. (2001). The language of new media. Cambridge: MIT Press.

Meier, W. (2003). eXist: An open source native XML database. In: A.B. Chaudhri et al. (Eds). Web Databases and Web Services 2002, LNCS 2593, 169 - 183.

Miller, G. A. \& Charles, W. G. (1991). Contextual correlates of semantic similarity. Languages and Cognitive Processes 6 (1), 1-28.

Miao, Y., Fleschutz, J. M. \& Zentel, P. (1999). Enriching learning contexts to support communities of practice. Proceedings of the 3rd International Conference on Computer Supported Collaborative Learning (CSCL), Stanford, USA, 391-397.

Motta, E. (1998). An overview of the OCML modelling language. Proceedings of KEML'98: 8th Workshop on Knowledge Engineering Methods \& Languages, Karlsruhe, Germany, Jan. 21-22.

Mylopoulos, J., Borgida, A., Jarke, M. \& Koubarakis, M. (1990). Telos - A language for managing knowledge about information systems. ACM Trans. Inform. Systems, 8 (4), 327-362.

Nelson, T. H. (1967). Getting it out of our system. In G. Schlechter (Ed.), Information Retrieval: A Critical Review. Washington, DC: Thompson Books.

Nelson, T. H. (1999). Xanalogical structure, needed now more than ever: Parallel documents, deep links to content, deep versioning, and deep re-use. ACM Computing Reviews, 33 (4).

Noy, N.F. \& Musen, M.A. (2002). PROMPTDIFF: A fixed-point algorithm for comparing ontology versions. Proceedings of the National Conference on Artificial Intelligence, 744-750.

Pfister, H.-R., Wessner, M., Holmer T. \& Steinmetz, R. (1999). Negotiating about shared knowledge in a cooperative learning environment. Proceedings of the 3rd International Conference on Computer Supported Collaborative Learning (CSCL), Stanford, USA, 454-457.

Porush, D. (1994). Educating the Hebrew brain. Katadion, Spring 1994.

Preston, J. A. \& Wilson, L. (2001). Offering CS1 on-line reducing campus resource demand while improving the learning environment. SIGCSE Bulletin, 33 (1), 342-346. 
Resnik, P. (1998). Semantic similarity in a taxonomy: An Information-Based Measure and its Application to Problems of Ambiguity in Natural Language. Journal of Artificial Intelligence Research, 11, 98130.

Reynolds, D. (2001). An RDF framework for resource discovery. In Decker, D. Fensel D., et al. (Eds.). Proceedings of the Second International Workshop on the Semantic Web-SemWeb 2001.

Rodriguez, A. \& Egenhofer, M.J. (2003). Determining semantic similarity among entity classes from different ontologies. IEEE Transactions on Knowledge and Data Engineering, 15, 442-456.

Rodriguez, F. G., Silva, J. L. P., Rosano, F. L., Contreras, F. C., \& Vitela, A. M. (2001). Creating a high school physics video-based laboratory. IEEE Multimedia, 8 (3), 78-86.

Salembier, P. \& Sikora, T. (Eds.) (2002). Introduction to MPEG-7 - Multimedia content description interface. John Wiley \& Sons.

Schmidt, J. W., Sehring, H.-W. \& Warnke, M. (2001). Der bildindex zur politischen ikonographie in der Warburg Electronic Library -Einsichten eines interdisziplinären Projektes. In Archivprozesse, (in German).

Schmitz, D. (2003). Adressierung digitaler Medien mittels Community-basierter Agententechnik. Proceedings of: Informatiktage, Bad Schussenried, Germany, November 8-9, (in German).

Schär, S.G. \& Krueger, H. (2000). Using new learning technologies with multimedia. IEEE Multimedia, 7 (3), 40-51.

Scollon, R. (1999). Mediated discourse and social interaction. Research on Language and Social Interaction, 32 (1\&2), 149-154.

Scott, J. (2000). Social network analysis - A handbook ( $2^{\text {nd }}$ ed.). London: Sage Publications.

Smith, B. \& Welty, C. (2001). Ontology: Towards a new synthesis. In C. Welty, \& B. Smith, (Eds.), Formal Ontology in Information System (pp. iii-x.). Ongunquit, Maine: ACM.

Smith, B., Welty, C. \& McGuinness, D. L. (2004). OWL web ontology language Guide. Retrieved from http://www.w3.org/TR/owl-guide/

Smith, B. K., Blankinship, E. \& Lackner, T. (2000). Annotation and education. IEEE Multimedia, 7 (2), 8489.

Sumner, T. \& Buckingham Shum, S. (1998). From documents to discourse: shifting conceptions of scholarly publishing. Conference on Computer-Human Interaction (CHI), 95-102.

Tversky, A. (1977). Features of similarity. Psychology Review, 84, 327-352.

Wagstaff, J. (2004). Wikipedia: It's wicked. The Wall Street Journal Online, Loose Wire. Retrieved from http://www.theproduct.com/6m105/readings/spring04/encyclopedia.pdf.

Westermann, U. \& Klas, W. (2003). An analysis of XML database solutions for the management of MPEG-7 media descriptions. ACM Computing Survey, 35 (4, December), 331-373.

Wenger, E. (1998). Communities of Practice: Learning, meaning, and identity. Cambridge University Press.

Wikipedia. (2004). Michel Foucault. Retrieved from http://en.wikipedia.org/wiki/Michel_Foucault

World Wide Web Consortium: Resource Description Framework (RDF) Model and Syntax Specification. (1999) Retrieved from http://www.w3.org/TR/REC-rdf-syntax/. 


\section{Biographies}

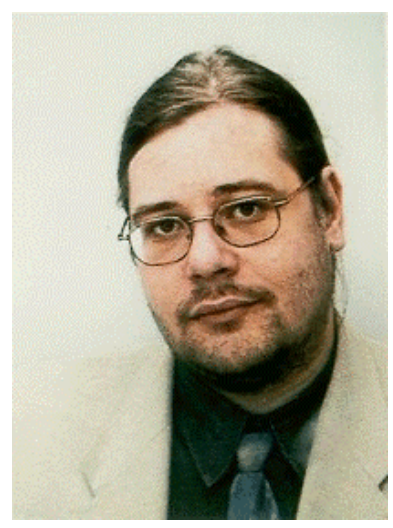

Ralf Klamma is a senior researcher within the Information Systems group at RWTH Aachen University. He has visited the Massachusetts Institute of Technology, Cambridge and has been a substitute professor at the universities of Chemnitz and Passau. He is project manager in the collaborative research center "Media and Cultural Communication" and core member of the European Network of Excellence PROLEARN in professional training. His research interests include theory and utilization of information systems, organizational memories and workflow management, virtual community support, electronic learning and professional training.

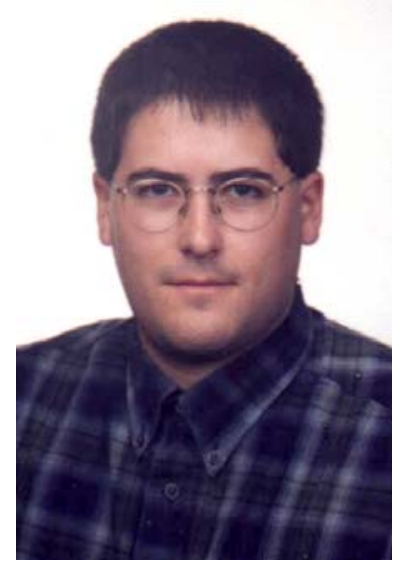

Marc Spaniol is a doctoral researcher in the Information Systems group at RWTH Aachen University. Within the Collaborative Research Center on "Media and Cultural Communication", he works on the project on "Impacts of Networked Multimedia Information Systems on Cooperation and Organization of Knowledge in Cultural Science Communities". He holds a diploma in computer science. His research interests include Multimedia Information Systems for Communities, Multimedia Metadata Standards such as MPEG-7, and Cross-Media Analysis.

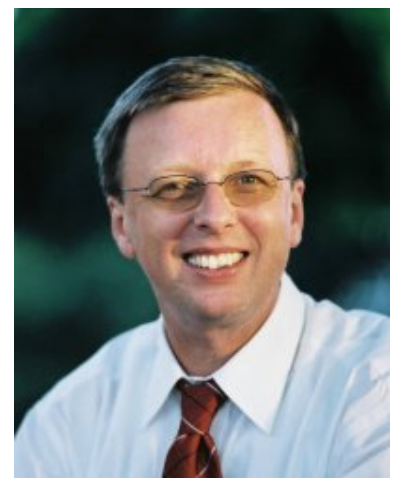

Matthias Jarke is Professor of Information Systems at RWTH Aachen University and Director of the Fraunhofer FIT Institute for Applied Information Technology in Sankt Augustin, Germany. Jarke studied Computer Science and Busienss Administration at the University of Hamburg and held faculty positions in New York University and the University of Passau prior to joining RWTH in 1991. His research interest is information systems support for cooperative applications in engineering, business, and culture. Jarke served a Chief Editor of Information Systems from 1993-2004 and is currently president of GI, the German Informatics society. 